\title{
High-order continuous and discontinuous Galerkin methods for wave problems
}

\author{
Giorgio Giorgiani, David Modesto, Sonia Fernández-Méndez and Antonio Huerta* \\ Laboratori de Calcul Numeric (LaCaN). Departament de Matematica Aplicada III \\ E.T.S. de Ingenieros de Caminos, Canales y Puertos, \\ Universitat Politecnica de Catalunya - BarcelonaTech, Jordi Girona 1, 08034 Barcelona, Spain. \\ web http://www.lacan.upc.edu
}

\begin{abstract}
SUMMARY
Three Galerkin methods - continuous Galerkin (CG), Compact Discontinuous Galerkin (CDG) and Hybridizable Discontinuous Galerkin (HDG) - are compared in terms of performance and computational efficiency in two-dimensional scattering problems for low and high-order polynomial approximations. The total number of degrees of freedom and the total runtime are used for this correlation as well as the corresponding precision. The comparison is carried out through various numerical examples. The superior performance of high-order elements is shown. At the same time, similar capabilities are shown for CG and HDG, when high-order elements are adopted, both of them clearly outperforming CDG.
\end{abstract}

Received ...

KEY WORDS: High-order elements, discontinuous Galerkin, wave propagation, scattering.

\section{INTRODUCTION}

Many engineering applications and physical phenomena, such as acoustic waves, electromagnetism and vibrations, are modeled with wave equations. The assumption of harmonic character of the solution leads to Helmholtz-type wave equations, with non-constant coefficients in the general case. Solving this problem for high frequencies leads to numerical difficulties because of the loss of the elliptic character and the oscillatory behavior of the solution. When tackling the problem with any finite element method, the characteristic size, $h$, of the spatial discretization has to be sufficiently small. Often, it is suggested to use constant $k h$, where $k$ is the wave number, see [1,2]. In practice, this means using a fixed number of nodes per wavelength (i.e. wave resolution) in each spatial direction. However, in [2] it is also shown that this rule guarantees an accurate solution only for low wave numbers. In fact, as shown in [3], the a priori error estimate in energy semi-norm of the finite element solution can be written as

$$
e_{h} \leq C_{1}\left(\frac{k h}{2 p}\right)^{p}+C_{2} k\left(\frac{k h}{2 p}\right)^{2 p}
$$

where $C_{1}$ and $C_{2}$ are two constants independent of $k$ and $h$, and $p$ is the polynomial degree of the approximation. The first term in (1) corresponds to the interpolation error. The second term is

*Correspondence to: Antonio Huerta, Laboratori de Càlcul Numèric (LaCàN), E.T.S. Ingenieros de Caminos, Universitat Politècnica de Catalunya, Jordi Girona 1, E-08034 Barcelona, Spain. 
the pollution error, which is strictly related with the difference in wavelength between the exact and the finite element solution, that is, the dispersion error, see $[4,5]$ for more details. For large wave numbers, the pollution error becomes the dominating term in (1) and it is responsible for the degradation of the finite element solution at a fixed wave resolution. Thus, equation (1) shows that increasing the wave number requires increasing the number of nodes per wavelength to keep a fixed level of accuracy.

Many techniques have been proposed in the past to reduce the dispersion error. Stabilized finite elements [6-15] is probably the most popular. Embedding the oscillatory behavior of the solution in the approximating functions is another option to improve accuracy, see for instance [16-23]. Another alternative, obvious from (1), is to increase the order of the approximation $p$, see [24]. This also coincides with recent results showing that high-order computations can be more efficient than low-order ones [25-31].

Discontinuous Galerkin methods [32-37] can also be an alternative in this area. In fact, [38,39] show a reduction in pollution errors. In [38] the interior penalty discontinuous Galerkin method performs better than CG in the pre-asymptotic range (large element size) for the Helmholtz equation. In [39], a discontinuous finite element formulation of the Helmholtz equation is proposed, which requires less degrees of freedom (DOF) than $\mathrm{CG}$ for properly chosen parameters. Note however that both references $[38,39]$ are restricted to linear approximations and, moreover, CG is hampered by the fact that no stabilization is used. To the authors knowledge there are no comparisons for highorder approximations between continuous and discontinuous Galerkin where pollution errors do not require to stabilize $\mathrm{CG}$.

Here CG and DG methods are compared in a two-dimensional (2D) scattering problem in a wide range of polynomial degrees. Two DG methods are studied: compact discontinuous Galerkin (CDG) [34] because it introduces the smallest stencil, as interior penalty [32], and hybridizable discontinuous Galerkin (HDG) [35-37,40] because it reduces the global solve to the DOF on the sides - similarly to CG with static condensation, see [41], and the Discontinuous Enriched Method [19] — and furthermore presents super-convergence properties.

The comparison is carried out through various numerical examples. Two measures of the computational cost are considered: the dimension of the final linear system, and the total runtime. On one hand, while the criterion based on the dimension of the linear system is implementation-free and gives also a measure of the hardware requirements, it does not take into account the structure of the linear system matrix, which can be significantly different for different methods and different polynomial interpolations. Moreover, the dimension of the linear system does not take into account the assembly cost, which is different for each Galerkin scheme.

On the other hand, the CPU time criterion takes into account all the costs, but obviously the results may depend on the actual implementation used for each method. To minimize this dependency, the same code optimization has been used for all methods, see [42,43] for details, and the same direct linear system solver has been used for all the computations. Note that this can be disadvantageous for DG methods because the structure of the information (constant bandwidth of block dense matrices) allows important gains in today's hardware, see for instance [41], and they are not exploited here.

The paper is organized as follows. Sections 2 and 3 are dedicated to the problem statement, and to recall basic concepts and assumptions on the three Galerkin methods that will be compared. Section 4 compares HDG, CG and CDG with three examples. First, an academic scattering problem with known analytical solution (the scattering of a plane wave against a circular object) is used to study, among others, cost at constant error level for the three methods at different $p$. Since CDG is not competitive, only CG and HDG are used in the following examples. The second case presents a geometry with corners, that induces a singular solution for the wave equation [44], which leads to possible convergence issues for high-order elements, see [45]. Finally, the comparison is extended to the computation of the wave propagation in the Barcelona harbor, corroborating the conclusions of the two previous academic tests. 


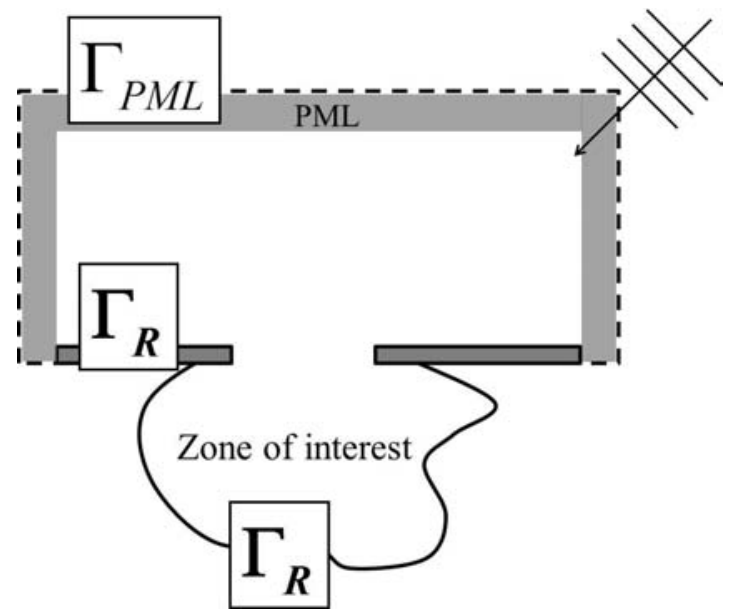

Figure 1. Problem statement.

\section{PROBLEM STATEMENT}

The following form of the Helmholtz equation is considered

$$
\nabla \cdot(\beta \nabla u)+k^{2} \beta u=\hat{f},
$$

in an unbounded $2 \mathrm{D}$ domain where $\beta(x, y) \in \mathbb{R}$ is a material parameter, $k(x, y) \in \mathbb{R}$ is the wave number, and $\hat{f}$ is an harmonic source. This equation models wave propagation for several problems of academic and engineering interest. One of the most challenging ones is the propagation of sea waves with a slowly-varying bottom depth. In this case, the coefficients are non-constant, large domains with small geometrical features induce very large systems of equations, two significant digits of accuracy are sought, and the number of runs can be very large due to the wide range of input data. Equation (2) is in this case also known as the Berkhoff or Mild Slope equation, see Section 4.3.

Boundary conditions are, on one hand, for reflecting/absorbing boundaries, $\Gamma_{R}$,

$$
\boldsymbol{n} \cdot \beta \boldsymbol{\nabla} u-I k \alpha \beta u=\hat{g} \text { on } \Gamma_{\mathrm{R}},
$$

where $I=\sqrt{-1}$ is the imaginary unit, $\hat{g}$ is a data function and $\alpha \in[0,1]$ is a real coefficient. This coefficient is equal to zero on totally reflecting boundaries and to one on perfectly absorbing boundaries, see for instance [46]. On the other hand, unbounded scattering problems require the so-called Sommerfeld radiation condition

$$
\lim _{r \rightarrow \infty} \sqrt{r}\left(\frac{\partial}{\partial r}-I k\right)\left(u-u_{0}\right)=0,
$$

where $r$ is the radial direction and $u_{0}$ the incident wave. The Sommerfeld radiation condition requires, in practice, the introduction of an artificial boundary and its corresponding boundary condition.

In this case it is usual to define a bounded computational domain and to introduce an artificial boundary, see among others $[47,48]$. Note that the coefficient $\beta$ is assumed constant at the artificial boundary and beyond, at least in the normal direction. Here, a Perfectly Matched Layer (PML) surrounds the computational domain in order to absorb outgoing waves, see for instance [49-52]. The setup of the problem is illustrated in Figure 1, showing the PML region. The problem to be solved is then

$$
\begin{aligned}
\boldsymbol{\nabla} \cdot(\beta \mathbf{P} \boldsymbol{\nabla} u)+k^{2} s_{x} s_{y} \beta u & =s_{x} s_{y} f & & \text { in } \Omega, \\
\boldsymbol{n} \cdot \beta \boldsymbol{\nabla} u-I k \alpha \beta u & =g & & \text { on } \Gamma_{\mathrm{R}}, \\
\boldsymbol{n} \cdot \beta \mathbf{P} \boldsymbol{\nabla} u-I k \beta u & =0 & & \text { on } \Gamma_{\mathrm{PML}},
\end{aligned}
$$


where $u$ is now the scattered field, $\Omega$ is the bounded computational domain including the PML, $f$ and $g$ are modifications of $\hat{f}$ and $\hat{g}$ accounting for the incident wave, $\mathbf{P}$ is the diagonal anisotropy matrix defining the absorption in the PML medium, namely

$$
\mathbf{P}=\left(\begin{array}{cc}
s_{y} / s_{x} & 0 \\
0 & s_{x} / s_{y}
\end{array}\right)
$$

and $s_{x}$ and $s_{y}$ are the absorption parameters in the two Cartesian directions. Note that $s_{x}=s_{y}=1$ outside the PML region. More details on the application of the PML to the Helmholtz equation can be found in [52]. Equation (4c) is a first order non-reflecting boundary condition discretizing (3) on $\Gamma_{\mathrm{PML}}$, to minimize non-physical reflection from the PML outer boundary. Thus, $\partial \Omega=\bar{\Gamma}_{\mathrm{R}} \cup \bar{\Gamma}_{\mathrm{PML}}$ with $\Gamma_{\mathrm{R}} \cap \Gamma_{\mathrm{PML}}=\emptyset$, and no Dirichlet boundary conditions are imposed.

\section{CONTINUOUS AND DISCONTINUOUS GALERKIN METHODS}

Let $\Omega \subset \mathbb{R}^{2}$ be the open computational bounded domain defined in Figure 1 with boundary $\partial \Omega$. Suppose that $\Omega$ is partitioned in $n_{e l}$ disjoint subdomains $\Omega_{i}$ with boundaries $\partial \Omega_{i}$. The following formal definitions and notation are used for the computation domain and its broken counterpart

$$
\bar{\Omega}=\bigcup_{i=1}^{\mathrm{n}_{\mathrm{e} 1}} \bar{\Omega}_{i}, \Omega_{i} \cap \Omega_{j}=\emptyset \text { for } i \neq j \text {, and } \widehat{\Omega}:=\bigcup_{i=1}^{\mathrm{n}_{\mathrm{e} 1}} \Omega_{i} .
$$

The union of all interior sides is

$$
\Gamma:=\bigcup_{\substack{i, j=1 \\ i \neq j}}^{\mathrm{n}_{\mathrm{el}}} \bar{\Omega}_{i} \cap \bar{\Omega}_{j}=\left[\bigcup_{i=1}^{\mathrm{n}_{\mathrm{e} 1}} \partial \Omega_{i}\right] \backslash \partial \Omega
$$

and the union of all $\mathrm{n}_{\mathrm{sd}}$ sides, $\Gamma_{i}$ for $i=1, \ldots, \mathrm{n}_{\mathrm{sd}}$, is $\Gamma^{+}:=\Gamma \cup \partial \Omega$. The mesh skeleton $\Gamma^{+}$has its broken counterpart denoted by $\widehat{\Gamma}^{+}$.

The previous discretization allows to define the finite dimensional spaces: $\mathcal{V}_{h}, \widehat{\mathcal{V}}_{h}$, and $\widehat{\Lambda}_{h}$, namely

$$
\begin{aligned}
& \mathcal{V}_{h}:=\left\{v \in \mathcal{H}^{1}(\Omega):\left.v\right|_{\Omega_{i}} \in \mathcal{P}^{p}\left(\Omega_{i}\right), \text { for } i=1, \ldots, \mathrm{n}_{\mathrm{el}}\right\}, \\
& \widehat{\mathcal{V}}_{h}:=\left\{v \in \mathcal{L}^{2}(\Omega):\left.v\right|_{\Omega_{i}} \in \mathcal{P}^{p}\left(\Omega_{i}\right), \text { for } i=1, \ldots, \mathrm{n}_{\mathrm{el}}\right\} \\
& \widehat{\Lambda}_{h}:=\left\{\mu \in \mathcal{L}^{2}\left(\Gamma^{+}\right):\left.\mu\right|_{\Gamma_{i}} \in \mathcal{P}^{p}\left(\Gamma_{i}\right), \text { for } i=1, \ldots, \mathrm{n}_{\mathrm{sd}}\right\},
\end{aligned}
$$

where $\mathcal{P}^{p}$ denotes the space of polynomials of degree $\leq p$. Note that $\mathcal{V}_{h}$ is a space of continuous functions in $\Omega$, whereas $\widehat{\mathcal{V}}_{h}$ and $\widehat{\Lambda}_{h}$ are discontinuous approximation spaces, corresponding to the broken domains $\widehat{\Omega}$ and $\widehat{\Gamma}^{+}$respectively.

Standard nodal basis are used to represent these spaces. In Figure 2 the nodes corresponding to a polynomial degree $p=5$ in a triangle are shown for the three spaces introduced. Fekete node distributions (for both, straight-sided and curved elements) are considered to minimize illconditioning, see [53] and [54, Sc. 4.3]. Obviously, under the ideal situation of exact arithmetic computations, the solution would be independent of the nodal distribution.

For functions with discontinuities between elements, the jump $\llbracket \cdot \rrbracket$ and the mean $\{\cdot\}$ operators are defined along the interface $\Gamma$ using values from the elements to the left and right of the interface (say, $\Omega_{i}$ and $\Omega_{j}$ ), namely

$$
\llbracket \odot \rrbracket=\odot_{i}+\odot_{j}, \quad\{\odot\}=\left(\odot_{i}+\odot_{j}\right) / 2 .
$$

The major difference between the mean and the jump operator is that the latter always involves the normal to the interface, see [55] for more details. 


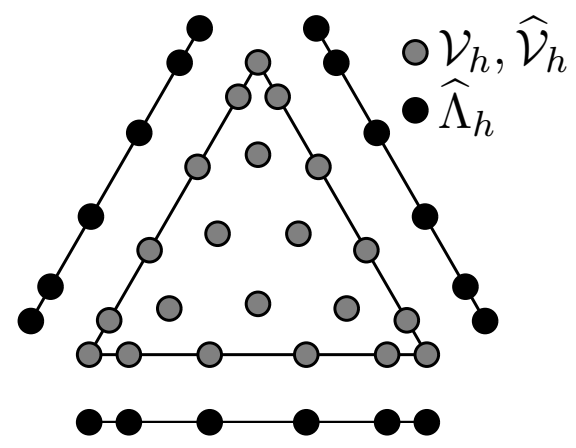

Figure 2. Nodes representing the spaces $\mathcal{V}_{h}, \widehat{\mathcal{V}}_{h}$, and $\widehat{\Lambda}_{h}\left(\Gamma^{+}\right)$.

\subsection{The continuous Galerkin formulation}

The standard discrete CG problem approximating (4) requires to find $u_{h} \in \mathcal{V}_{h}$ such that

$$
a\left(u_{h}, v\right)=l(v) \quad \forall v \in \mathcal{V}_{h},
$$

where the bilinear and linear forms, $a(\cdot, \cdot)$ and $l(\cdot)$ are given by

$$
\begin{gathered}
a(u, v)=(\beta \mathbf{P} \nabla u, \nabla v)_{\Omega}-(\gamma u, v)_{\Omega}-I\langle k \alpha \beta u, v\rangle_{\Gamma_{\mathrm{R}}}-I\langle k \beta u, v\rangle_{\Gamma_{\mathrm{PML}}}, \\
l(v)=\langle g, v\rangle_{\Gamma_{\mathrm{R}}}-\left(s_{x} s_{y} f, v\right)_{\Omega}
\end{gathered}
$$

with $\gamma:=k^{2} s_{x} s_{y} \beta$. In the previous and the following equations, $(\cdot, \cdot)_{D}$ denotes the $\mathcal{L}^{2}$ scalar product in any domain $D$, while $\langle\cdot, \cdot\rangle_{B}$ also denotes the $\mathcal{L}^{2}$ scalar product of the traces over $B$.

Note that high-order CG is always implemented using static condensation for the interior nodes of each element. This reduces considerably the number of global unknowns at an element-by-element cost of generating and solving the small Shur complement system of equations.

\subsection{The compact discontinuous Galerkin formulation}

The CDG formulation requires first to rewrite (4a) as a system of first order equations taking into account the discontinuities of the approximation spaces between elements. Thus equations (4) become:

$$
\begin{aligned}
\boldsymbol{\sigma}-\beta \mathbf{P} \boldsymbol{\nabla} u & =\mathbf{0} & & \text { in } \widehat{\Omega}, \\
\boldsymbol{\nabla} \cdot \boldsymbol{\sigma}+\gamma u & =s_{x} s_{y} f & & \text { in } \widehat{\Omega}, \\
\llbracket \boldsymbol{\sigma} \cdot \boldsymbol{n} \rrbracket & =0 & & \text { on } \Gamma, \\
\llbracket u \boldsymbol{n} \rrbracket & =\mathbf{0} & & \text { on } \Gamma, \\
\boldsymbol{n} \cdot \beta \boldsymbol{\nabla} u-I k \alpha \beta u & =g & & \text { on } \Gamma_{\mathrm{R}}, \\
\boldsymbol{n} \cdot \beta \mathbf{P} \nabla u-I k \beta u & =0 & & \text { on } \Gamma_{\mathrm{PML}} .
\end{aligned}
$$

As usual in CDG [34], two local lifting operators are defined on all the interior sides (recall that here no Dirichlet boundary conditions are applied). For any interior side $\Gamma_{e} \subset \Gamma$, the lifting $\boldsymbol{r}^{e}:\left[\mathcal{L}^{2}\left(\Gamma_{e}\right)\right]^{2} \rightarrow\left[\widehat{\mathcal{V}}_{h}\right]^{2}$ is defined by

$$
\left(\boldsymbol{r}^{e}(\boldsymbol{\sigma}), \boldsymbol{\omega}\right)_{\Omega}=\langle\boldsymbol{\sigma},\{\boldsymbol{\omega}\}\rangle_{\Gamma_{e}} \forall \boldsymbol{\omega} \in\left[\widehat{\mathcal{V}}_{h}\right]^{2}
$$

Likewise, the second lifting, $s^{e}: \mathcal{L}^{2}\left(\Gamma_{e}\right) \rightarrow\left[\widehat{\mathcal{V}}_{h}\right]^{2}$, is defined by

$$
\left(\boldsymbol{s}^{e}(v), \boldsymbol{\omega}\right)_{\Omega}=\langle v, \llbracket \boldsymbol{n} \cdot \boldsymbol{\omega} \rrbracket\rangle_{\Gamma_{e}} \quad \forall \boldsymbol{\omega} \in\left[\widehat{\mathcal{V}}_{h}\right]^{2},
$$

for all interior sides $\Gamma_{e} \subset \Gamma$. 
Following the rationale detailed in [34], the CDG weak form associated to (6) requires to find $u_{h} \in \widehat{\mathcal{V}}_{h}$ such that

$$
\hat{a}\left(u_{h}, v\right)=\hat{l}(v) \quad \forall v \in \widehat{\mathcal{V}}_{h},
$$

where the bilinear and linear forms, $\hat{a}(\cdot, \cdot)$ and $\hat{l}(\cdot)$ are given by

$$
\begin{aligned}
& \hat{a}(u, v)=(\beta \mathbf{P} \boldsymbol{\nabla} u, \boldsymbol{\nabla} v)_{\Omega}-(\gamma u, v)_{\Omega}-\langle\beta \mathbf{P} \llbracket u \boldsymbol{n} \rrbracket,\{\boldsymbol{\nabla} v\}\rangle_{\Gamma}-\langle\beta \mathbf{P}\{\boldsymbol{\nabla} u\}, \llbracket v \boldsymbol{n} \rrbracket\rangle_{\Gamma} \\
&-I\langle k \alpha \beta u, v\rangle_{\Gamma_{\mathrm{R}}}-I\langle k \beta u, v\rangle_{\Gamma_{\mathrm{PML}}}+\left\langle C_{11} \llbracket u \boldsymbol{n} \rrbracket, \llbracket v \boldsymbol{n} \rrbracket\right\rangle_{\Gamma} \\
&-\left\langle\beta\left(\mathbf{P} \boldsymbol{C}_{12}\right) \cdot \llbracket u \boldsymbol{n} \rrbracket, \llbracket \boldsymbol{n} \cdot \boldsymbol{\nabla} v\right\rangle_{\Gamma}-\left\langle\beta\left(\mathbf{P} \boldsymbol{C}_{12}\right) \cdot \llbracket v \boldsymbol{n} \rrbracket, \llbracket \boldsymbol{n} \cdot \boldsymbol{\nabla} u \rrbracket\right\rangle_{\Gamma} \\
&+\sum_{\Gamma_{e} \subset \Gamma}\left(\boldsymbol{r}^{e}(\beta \mathbf{P} \llbracket u \boldsymbol{n} \rrbracket)+\boldsymbol{s}^{e}\left(\beta\left(\mathbf{P} \boldsymbol{C}_{12}\right) \cdot \llbracket u \boldsymbol{n} \rrbracket\right), \boldsymbol{r}^{e}(\llbracket v \boldsymbol{n} \rrbracket)+\boldsymbol{s}^{e}\left(\boldsymbol{C}_{12} \cdot \llbracket v \boldsymbol{n} \rrbracket\right)\right)_{\Omega}, \\
& \hat{l}(v)=\langle g, v\rangle_{\Gamma_{\mathrm{R}}}-\left(s_{x} s_{y} f, v\right)_{\Omega} .
\end{aligned}
$$

Note that in the previous expressions differential operators are assumed to act on these functions piecewise (element-by-element) and not in the sense of distributions.

The CDG forms depend on two parameters, $C_{11}$ and $C_{12}$. The former, $C_{11}$, is a non-negative parameter of order $\mathcal{O}\left(h^{-1}\right)$ that, in absence of Dirichlet boundary conditions, may be considered $C_{11}=0$ on $\Gamma^{+}$, see [34]. The latter is an additional vector, $C_{12} \in \mathbb{R}^{2}$, which is defined for each interior side of the domain according to

$$
\boldsymbol{C}_{12}=\frac{1}{2}\left(S_{i j} \boldsymbol{n}_{i}+S_{j i} \boldsymbol{n}_{j}\right)
$$

where $S_{j i} \in\{0,1\}$ denotes the switch associated to element $\Omega_{i}$ on the side that element $\Omega_{i}$ shares with element $\Omega_{j}$. There are several possible choices of the switches, always satisfying $S_{i j}+S_{j i}=1$, see $[34,56]$ for details. Here the so-called consistent switch has been used [34].

Remark 1. Lifting operators in CDG are associated to individual sides, and therefore there are no connectivities between non-neighbor elements. This induces small stencils as in interior penalty methods, see the comparison in [57]. However, the connectivity between the interior nodes of one element and interface unknowns of neighboring elements, precludes the possibility of static condensation in CDG. See Remark 2 for a concise comparison of stencils and matrix structure of $\mathrm{CDG}$ and the other discussed methods.

\subsection{The hybridizable discontinuous Galerkin formulation}

Following [35], the HDG method for system (4) or, more precisely, for (6) can be stated as: find $\left(\sigma_{h}, u_{h}, \lambda\right) \in\left[\widehat{\mathcal{V}}_{h}\right]^{2} \times \widehat{\mathcal{V}}_{h} \times \widehat{\Lambda}_{h}$ such that

$$
\begin{gathered}
\left(\mathbf{Q} \boldsymbol{\sigma}_{h}, \boldsymbol{\omega}\right)_{\Omega_{i}}+\left(u_{h}, \boldsymbol{\nabla} \cdot \boldsymbol{\omega}\right)_{\Omega_{i}}-\langle\lambda, \boldsymbol{\omega} \cdot \boldsymbol{n}\rangle_{\partial \Omega_{i}}=0 \\
\left.\begin{array}{r}
\left.\boldsymbol{\nabla} \cdot \boldsymbol{\sigma}_{h}, v\right)_{\Omega_{i}}+\left(\gamma u_{h}, v\right)_{\Omega_{i}}-\left\langle\tau\left(u_{h}-\lambda\right), v\right\rangle_{\partial \Omega_{i}}=\left(s_{x} s_{y} f, v\right)_{\Omega_{i}}
\end{array}\right\} \text { for } i=1, \ldots, \mathrm{n}_{\mathrm{el}}, \\
\sum_{i=1}^{\mathrm{n}_{\mathrm{e} 1}}\left\langle\left(\boldsymbol{\sigma}_{h} \cdot \boldsymbol{n}-\tau\left(u_{h}-\lambda\right)\right), \mu\right\rangle_{\partial \Omega_{i}}-I\langle k \alpha \beta \lambda, \mu\rangle_{\Gamma_{\mathrm{R}}}-I\langle k \beta \lambda, \mu\rangle_{\Gamma_{\mathrm{PML}}}=\langle g, \mu\rangle_{\Gamma_{\mathrm{R}}},
\end{gathered}
$$

for all $(\boldsymbol{\omega}, v, \mu) \in\left[\widehat{\mathcal{V}}_{h}\right]^{2} \times \widehat{\mathcal{V}}_{h} \times \widehat{\Lambda}_{h}$, where a new matrix is defined $\mathbf{Q}:=(\beta \mathbf{P})^{-1}$. The trace variable $\lambda \in \widehat{\Lambda}_{h}$, which is single valued in each side of the mesh, is an approximation of the trace of the solution $u$ on the mesh sides.

The coefficient $\tau$ is a positive stabilization parameter, whose influence on the convergence properties of the HDG method has been studied in $[35,41,58]$. It may be prescribed as a positive value on every side of each triangular element (all faces approach) [41,58], or may be set to zero except on a single arbitrary chosen side of each element (single face approach) [35]. Both options require $\tau$ to be large enough for stability, and both provide, for properly chosen values of $\tau$, optimal convergence in the solution $\eta$ and its gradient $\boldsymbol{\sigma}$, viz. order $p+1$ in $\mathcal{L}_{2}$ norm. More precisely, 
extensive numerical evidence $[35,41]$ shows that values of order one provide optimal behavior for a dimensionless problem. Nevertheless, the single face approach presents an increased robustness for the choice of $\tau$, it allows using larger values without any remarkable impact in the solution. This is not the case for the all faces approach. When large values of $\tau$ are prescribed in every side superconvergence of the post-processed solution can be lost. Note that this behavior induces an extra difficulty for equations with non-constant coefficients, such as (2), because $\tau$ should vary in each element to account for the variability of the coefficients. In conclusion, the single face stabilization parameter is considered here, that is

$$
\tau=\left\{\begin{array}{ll}
0 & \text { on } \partial \Omega_{i} \backslash \partial \Omega_{i}^{\tau} \\
\tau_{i} & \text { on } \partial \Omega_{i}^{\tau}
\end{array} \text { for } i=1, \ldots, \mathrm{n}_{\mathrm{el}},\right.
$$

with a constant $\tau_{i}>0$ and $\partial \Omega_{i}^{\tau}$ an arbitrary but fixed side of element $i$. A constant value $\tau_{i}$ is used for all the elements, and a simple dimensional analysis leads to the following expression for the minimum value of $\tau_{i}$ to be used

$$
\tau_{i} \geq \tau^{-}:=\max _{\Omega}\left\{k \beta\left|s_{x} / s_{y}\right|\right\} \text { for } i=1, \ldots, \mathrm{n}_{\mathrm{el}},
$$

which would correspond to $\tau^{-} \approx 1$ for a dimensionless problem with constant coefficients. Note that $\tau$ is defined element-by-element, thus a side shared by two elements may have two different values of $\tau$.

Note that, in contrast to other mixed or hybrid methods, HDG allows for equal interpolation in the space of the primal unknown, $\widehat{\mathcal{V}}_{h}$, and the space of its gradient, $\left[\widehat{\mathcal{V}}_{h}\right]^{2}$, as well as for the trace variable, $\widehat{\Lambda}_{h}$. This is due to the particular form of the numerical fluxes and the stabilization parameter $\tau$. They ensure solvability and stability, see [35], without the need of an enriched space for the gradient variable, or a reduced space for the trace variable.

From a computational point of view, there are $n_{e l}$ local problems induced by (7a), which correspond to solving all the Schur complements (static condensation) for the interior nodes in CG. The global solve in HDG, which computes the trace $\lambda$, follows from (7b).

Remark 2 (Stencil and sparsity). At this point it is possible to compare the stencils, the matrix sparsity and the number of DOF for CG, HDG and CDG. The smallest global solve induced by these methods always corresponds to CG because there are no interior nodes (this is not the case of $\mathrm{CDG}$ ) and there is no node multiplicity. Figure 3 shows the nodes involved in a global solve for the three methods on a simple mesh. Note that HDG, which also avoids interior nodes, has more DOF compared to CG because vertices have a multiplicity equal to the number of sides connected to them. Nevertheless, the weight of the multiplicity of the vertices decreases as the approximation $p$ is increased. Moreover, Figure 3 also depicts the stencils induced by vertex and side nodes. Side nodes have, more or less, similar foot-prints (bottom plots in Figure 3) but vertex nodes present substantial differences. In particular it is worth observing that the larger foot-print is induced by CG.

From a computational viewpoint DG methods present another advantage that is depicted in Figure 4: their uniform block structure. This figure shows the matrix sparsity pattern for CG, HDG and $\mathrm{CDG}$, and the meshes shown Figure 3 with degree $p=3$. For a structured mesh such as the one presented in Figure 3 but, more important, for an unstructured mesh also, the interconnection is always constant. For instance, in HDG each face is always connected to four neighbor faces. Thus, any node is only connected to the nodes in the four neighbor faces (always the same amount). This is not the case a vertex node in CG in an unstructured mesh.

These advantages of the uniform block structure induced by HDG become more apparent for high-order computations. For example, HDG is $10 \%$ faster than CG in the runtime required by the global solve (direct solver) for the scattering circle of Section 4.1 with degree $p=9$. Note, that as mentioned earlier, HDG has more DOF than CG on the same mesh, namely 781226 for CG and 937230 for HDG. But the uniform block structure of HDG can be exploited by a standard reverse Cuthill-McKee reordering and produce smaller a bandwidth, namely 5161 for CG and 3438 for HDG. Dedicated block solvers for HDG could further improve this performance thanks to the 

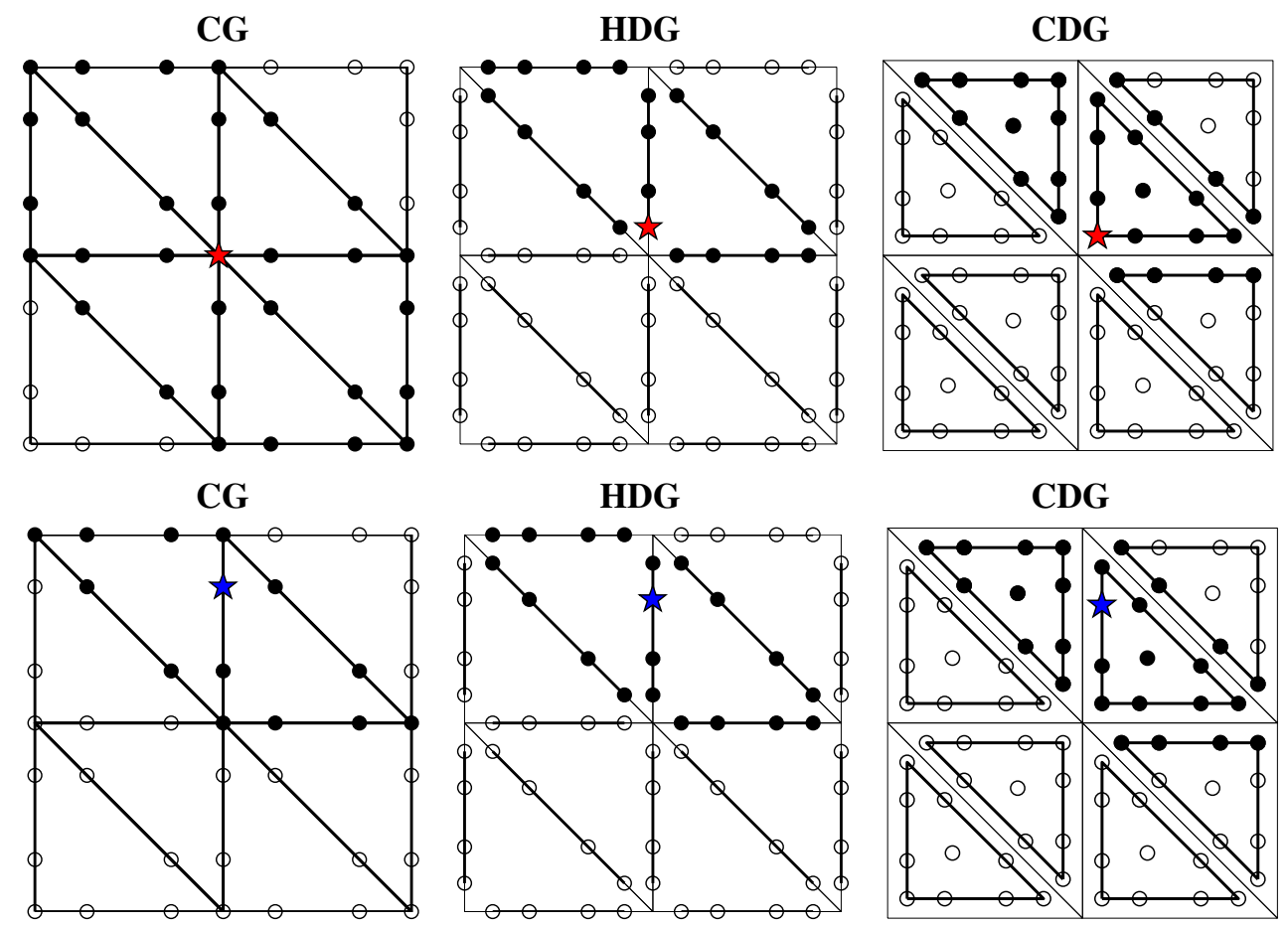

Figure 3. Stencil for a vertex node (top, red star) and a side node (bottom, blue star) for a CG, HDG and CDG discretization. For CG and HDG, only the nodes corresponding to degrees of freedom, after static condensation, are depicted. Nodes connected to the node marked with a star are marked as solid black dots.
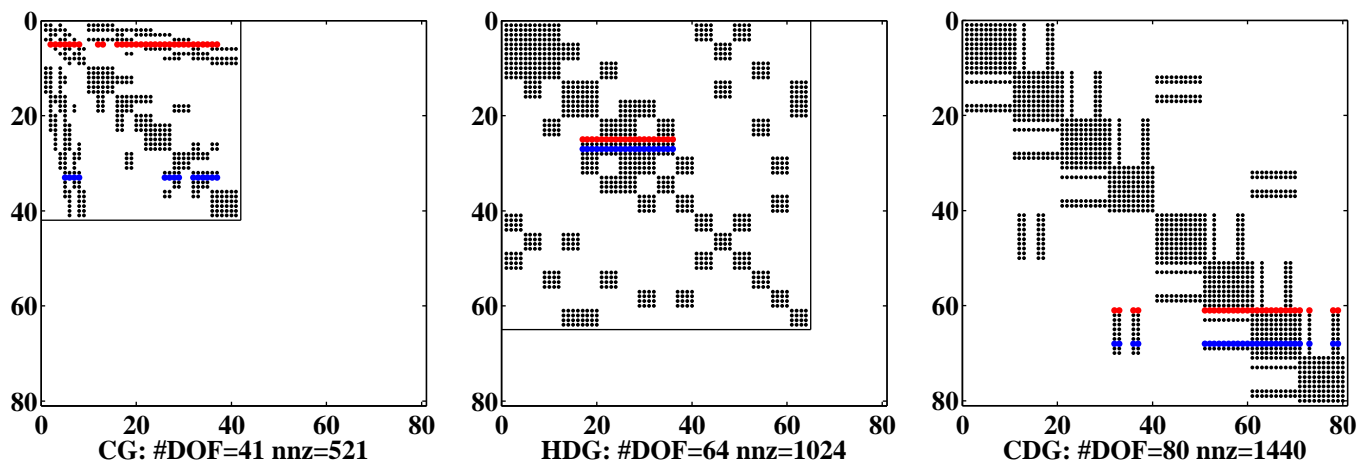

Figure 4. Sparsity pattern of the matrix induced by the mesh shown in Figure 3 for CG, HDG and CDG, with degree $p=3$. The equations (rows) corresponding to the nodes marked with stars in Figure 3 are depicted with in red, for the vertex node, and in blue, for the side node. The system dimension (\#DOF) and the number of non-zero entries (nnz) are listed for each method.

uniformity in the structure. These results are in accordance with [41] where the solver efficiency, comparing HDG and CG on the same mesh with degree $p>5$ is presented.

Remark 3 (HDG post-processed solution). With the solution of the global problem described by (7b), the $\mathrm{n}_{\mathrm{el}}$ local problems (7a) give an approximation of $\left(u_{h}, \sigma_{h}\right) \in \widehat{\mathcal{V}}_{h} \times\left[\widehat{\mathcal{V}}_{h}\right]^{2}$. Then, for each element, equation (6a) can be solved using the corresponding Neumann boundary conditions and a 
Table I. Order of the quadrature rules employed with their corresponding number of integration points.

\begin{tabular}{lrrrrrrrrr}
\hline Order of quadrature rule & 2 & 3 & 5 & 6 & 10 & 15 & 20 & 25 & 30 \\
\hline Number of integration points & 3 & 4 & 7 & 12 & 25 & 85 & 85 & 126 & 175 \\
\hline
\end{tabular}

restriction for solvability, namely

$$
\left.\begin{array}{rlrl}
-\boldsymbol{\nabla} \cdot \boldsymbol{\nabla} u^{*} & =\boldsymbol{\nabla} \cdot\left(\mathbf{Q} \boldsymbol{\sigma}_{h}\right) & & \text { in } \Omega_{i} \\
-\boldsymbol{n} \cdot \boldsymbol{\nabla} u^{*} & =\boldsymbol{n} \cdot \mathbf{Q} \boldsymbol{\sigma}_{h} & & \text { on } \partial \Omega_{i} \\
\int_{\Omega_{i}} u^{*} d \Omega & =\int_{\Omega_{i}} u_{h} d \Omega &
\end{array}\right\} \text { for } i=1, \ldots, \mathrm{n}_{\mathrm{el}} .
$$

This induces a weak problem in a richer finite dimensional space, that is, find $u_{h}^{*} \in \widehat{\mathcal{V}}_{h}^{*}$ such that

$$
\left(\boldsymbol{\nabla} u_{h}^{*}, \nabla v\right)_{\Omega_{i}}=\left(\mathbf{Q} \sigma_{h}, \nabla v\right)_{\Omega_{i}} \text { and }\left(u_{h}^{*}, 1\right)_{\Omega_{i}}=\left(u_{h}, 1\right)_{\Omega_{i}},
$$

for all $v \in \widehat{\mathcal{V}}_{h}^{*}$ and $i=1, \ldots, \mathrm{n}_{\mathrm{el}}$. Where $\widehat{\mathcal{V}}_{h}^{*}$ must be a bigger space than $\widehat{\mathcal{V}}_{h}$. In fact, with one degree more in the element-by-element polynomial approximation, i.e. $\widehat{\mathcal{V}}_{h}^{*}=\left\{v \in \mathcal{L}^{2}(\Omega):\left.v\right|_{\Omega_{i}} \in\right.$ $\mathcal{P}^{p+1}\left(\Omega_{i}\right)$, for $\left.i=1, \ldots, \mathrm{n}_{\mathrm{el}}\right\}, u_{h}^{*}$ converges asymptotically at a rate of $p+2$ in the $\mathcal{L}^{2}$ norm, see [35].

\section{NUMERICAL RESULTS}

Three examples are used to compare the performance of low and high-order CG, CDG and HDG. The first one has an analytical solution allowing for a thorough error analysis. The others, which include a real engineering application, use a reference overkilled solution (high-order CG with a refined mesh) to evaluate the precision of the different approximations. Note that in all these comparisons the PML region is kept unmodified to minimize its influence on the analysis. Moreover, when comparing HDG the super-convergent solution described in Remark 3 is used.

To compare the performance of the different Galerkin methods two basic criteria are employed: the number of DOF (more precisely, the size of the global system to be solved) and the total runtime. These comparisons are done taking into account the precision of the results, the element characteristic size $h$, and the degree of the approximation $p$, which can be combined in the dimensionless wave number, see Remark 4.

Obviously, the computational time strongly depends, among others, on the quadrature rules employed. Here, Wandzurat symmetric rules for triangles are considered, see [59]. Moreover, in order not to penalize low-order elements, some Dunavant rules for low orders are also implemented see [60]. Table I specifies the number of integration points corresponding to each order of the quadrature employed in the following examples. In practice, given the required integration order defined by the corresponding weak form and the degree of approximation $p$, the rule adopted is the lowest possible of those listed in Table I. For orders of quadratures larger than 30 no Wandzurat quadrature is available, and a tensor quadrature is used in this case.

Remark 4 (dimensionless wave number). The so-called dimensionless wave number $\xi:=k h / p$, where $h$ is the element size, incorporates the degree of the approximation $p$ and will be used to quantify the wave resolution. For example, $\xi=\pi / 4$ corresponds to 8 linear elements per wavelength and it is considered a minimum resolution for linear elements [61]. However, as already noted in [1] high-order elements require less nodes per wavelength.

\subsection{Homogeneous circular scattering of a plane wave}

A standard benchmark test for wave problems is considered [62]: a plane wave is scattered by a cylindrical object of unitary radius, $\mathrm{R}=1$, in a homogeneous media. In this case, the problem defined 

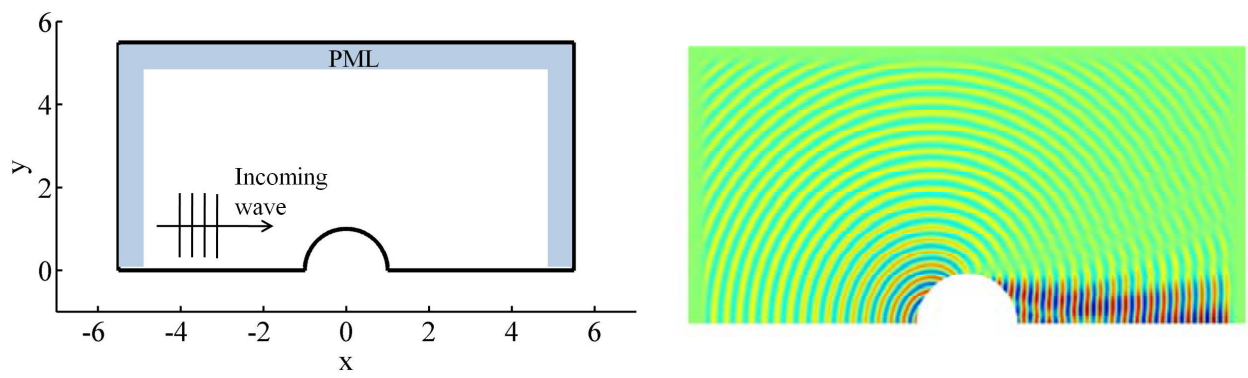

Figure 5. Circular scattering of a plane wave: problem statement (left) and scattered wave for $k=25$ (right).

in (4) is simplified because $\beta$ is constant and $f=0$. Moreover, given the symmetry of the problem, only half of the geometry is simulated as depicted in Figure 5. An analytical solution to this problem can be found in [63].

A study of the accuracy of the approximate solution is performed using unstructured triangular meshes for CG, CDG and HDG, and three different values of the wave number, $k=11,25$, and 100 , corresponding to a number of waves in the computational domain equal to 17,40 and 160 respectively. Note that this implies that the number of wavelengths per diameter of the obstacle ranges from $11 / \pi$ to $100 / \pi$. Thus, the range considered is in the mid-high frequency regime. As an example of the obtained results, Figure 5 depicts the solution for the middle wavelength: $k=25$. Four different values of the polynomial degree $p$ are tested, $p=1,2,5$, and 9 . For each combination of $k$ and $p$, four computations are carried out for each method, with mesh sizes corresponding to the dimensionless wave numbers $\xi=0.5,0.75,1$, and 1.25 , see Remark 4 . The corresponding wave resolutions are $12.6,8.4,6.3$, and 5.0 nodes per wavelength. Thus, a total of 144 simulations have been carried out. Note that for a given $\xi$ and wave number $k$, the meshes corresponding to different $p$ have approximately the same number of nodes in the domain.

Figure 6 depicts the relative $\mathcal{L}^{2}$ error as a function of the square root of the number of DOF (inversely proportional to the element size), for CG, CDG and HDG and the two extreme values: $k R=11$ and $k R=100$. The relative $\mathcal{L}^{2}$ error is evaluated over the computational domain (top) —not including the PML region — and on the scattering boundary (bottom). The mean slope of the curve is displayed at the bottom of each curve.

Low frequencies have convergence rate dominated by the interpolation error, i.e. first term in Equation (1). However for large wave numbers (high frequencies) and high-order approximations, it is clear that the slopes of the convergence curves are influenced by dispersion error, which increased the rate of convergence. On the contrary, discontinuous low-order approximations seem less sensible to dispersion, which is conforming with the results in $[38,39]$ for the interior penalty discontinuous Galerkin.

It is important to note that Figure 6 indicates that HDG always induces smaller errors than $\mathrm{CG}$ in the interior and on the scattering boundary on a given discretization (same $h$ and $p$ ). This is not the case for CDG at $p=9$.

These curves also show the efficiency of high-order computations: high-order approximations provide better accuracy than lower order approximations for the same number of DOF, i.e for the same size of the linear system. Equivalently, high-order approximations require less DOF to reach a given level of accuracy.

The number of DOF for a fixed value of the error is depicted in Figure 7. The number of DOF for each accuracy level is estimated from the convergence curves in Figure 6 and additional computations with $k=25$. More specifically, the number of DOF is linearly interpolated in log-log scale, when the chosen error level is in between the limits of the convergence curve, or it is linearly extrapolated using the closest two points.

The curves in Figure 7 also illustrate how an increase in $p$ reduces the number of DOF for a given precision for every method. This is in perfect agreement with $[1,3]$. Note that for a given frequency 

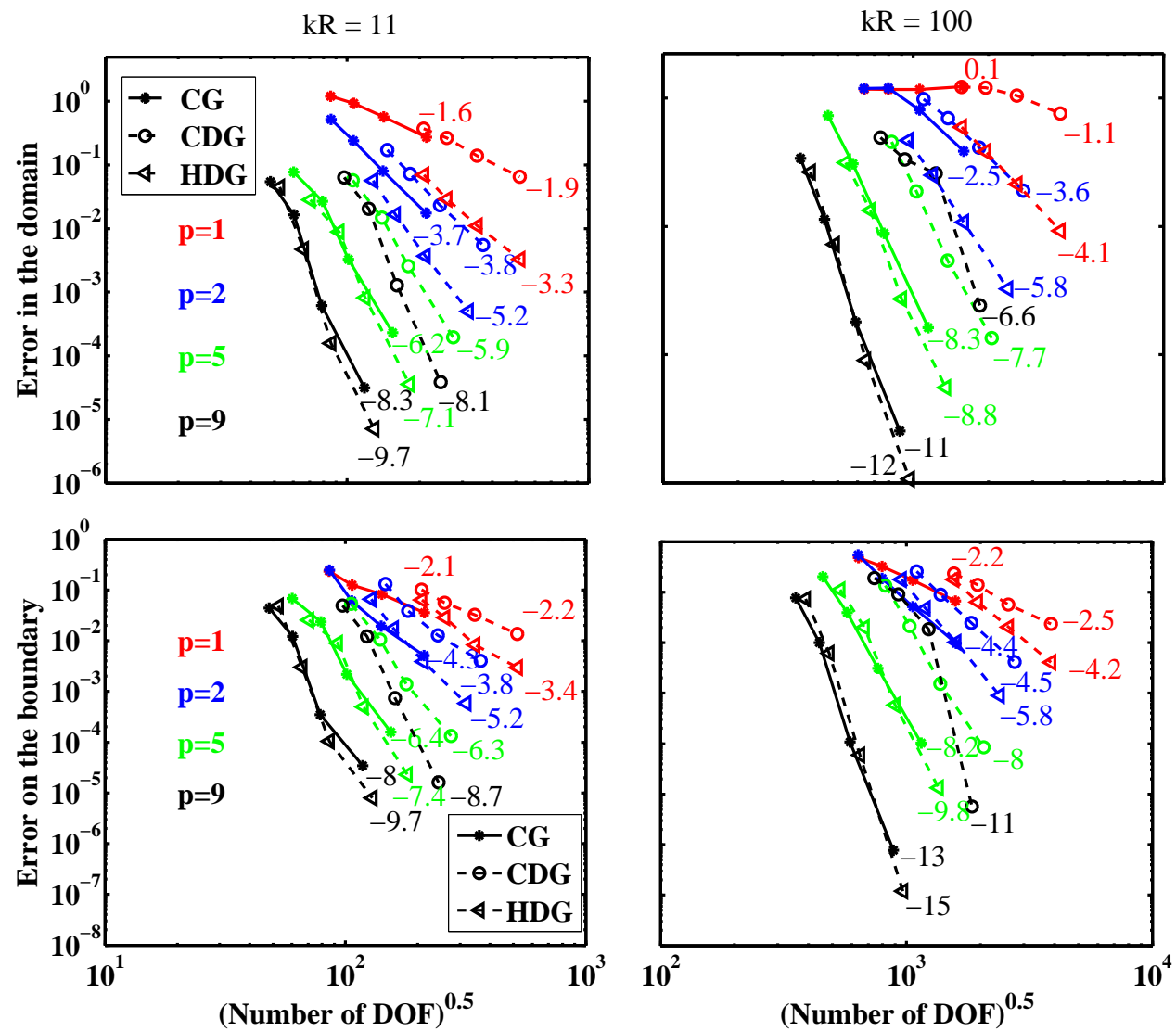

Figure 6. Circular scattering of a plane wave: error versus number of DOF for two different values of wave length $k R=11$ (left) and $k R=100$ (right) and over the computational domain (top) or the scattering boundary (bottom).
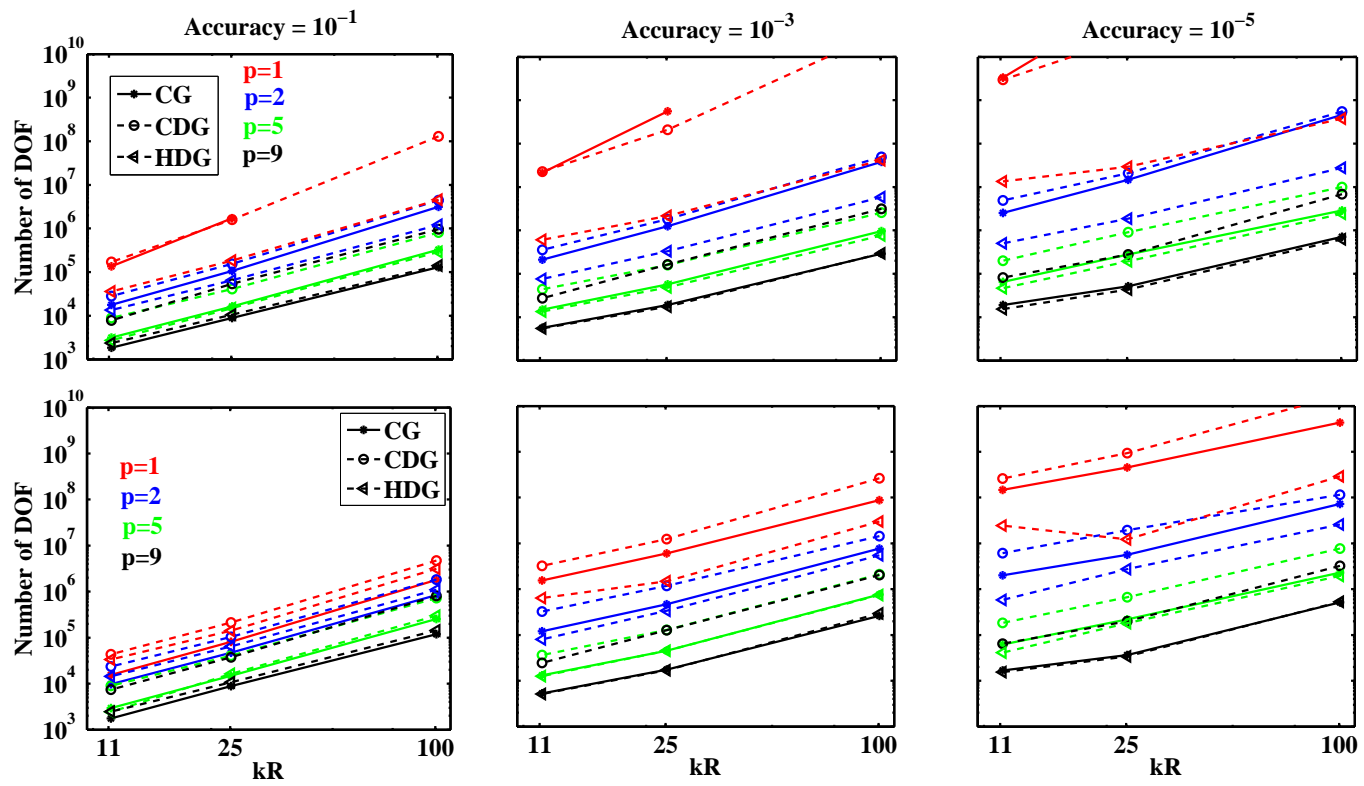

Figure 7. Circular scattering of a plane wave: DOF for an accuracy of 1e-1 (left), 1e-3 (center), 1e-5 (right), for the error in the domain (top) and on the scattering boundary (bottom). 

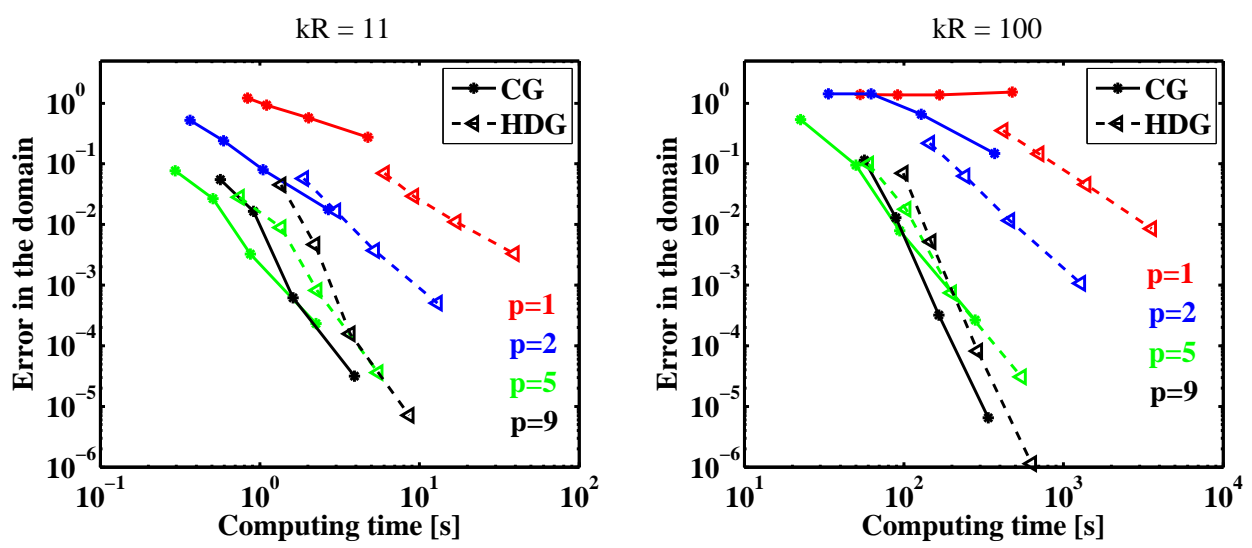

Figure 8. Circular scattering of a plane wave: error in the domain vs runtime for CG and HDG and $k R=11$ (left) and $k R=100$ (right).

the reduction in number of DOF is of several orders of magnitude for mid to high accuracies. Similar conclusions are inferred analyzing the error in the domain or the error on the scattering boundary.

For CG and HDG, the reduction in number of DOF for increasing $p$ is more important because of their lack of interior nodes. In fact, due to the similarity between static condensation and the hybridization technique, CG and HDG perform similarly, with almost the same number of DOF for a given accuracy level for high-order approximations. For low-order approximations $p=1,2$, HDG method performs better than CG without stabilization, due to the fact that DG methods are less sensitive to dispersion errors.

As expected, Figure 7 also shows that CDG requires considerable more DOF than HDG or CG for the same level of accuracy, due to the coupling of the nodes in the interior of the element with neighboring elements. Thus, the computational cost for the linear system solution is clearly larger for CDG than for CG or HDG. In fact, the computational cost for the linear system solution is related not only to the dimension of the linear system but also with the number of non-zero entries of the linear system matrix. These two factors penalize CDG.

To conclude, given that CG and HDG exhibit similar efficiency for high order elements, clearly outperforming CDG, CDG is discarded and will not be considered in the following tests, focusing the discussion in $\mathrm{CG}$ and HDG.

Next, CG and HDG are further compared in terms of runtime. The total time to obtain the solution is considered: matrix generation and assembly, linear system solution, and the evaluation of the solution at the interior nodes in CG and HDG. In this latter case the post-process for obtaining the super-convergent approximation, see Remark 3, is also taken into account.

As noted earlier the assembly time is largely influenced by the quadrature rules implemented. The order of the quadrature rules considered are those needed to exactly integrate a mass matrix of a straight-sided element, which corresponds to the second term in (4a) and, for this example, with constant coefficients. The overhead due to the integration on curved elements and PML elements (for which non-constant coefficients are present) is neglected, given that curved elements and PML elements are a small percent of the whole element set. For a given $p$, the quadrature rule adopted is the one with the lowest order that is $\geq 2 p$ in Table I.

All computations are carried out with Matlab running on a Xeon E5640, 2.66 Ghz/12MB cache with 72 GB of RAM. A code optimization based on the substitution of the code loops by matrixmatrix multiplication (whenever possible) has been adopted, see [42,43] for a detailed description of this optimization. For the linear system solution, a direct solver is considered. In contrast with the comparison in [41] done with an Hermitian positive definite matrix, here the complex matrix is symmetric but not Hermitian because of the boundary term in (5) and (7) and the complex coefficients in the PML region. Thus, a general LU factorization with partial pivoting is used. 

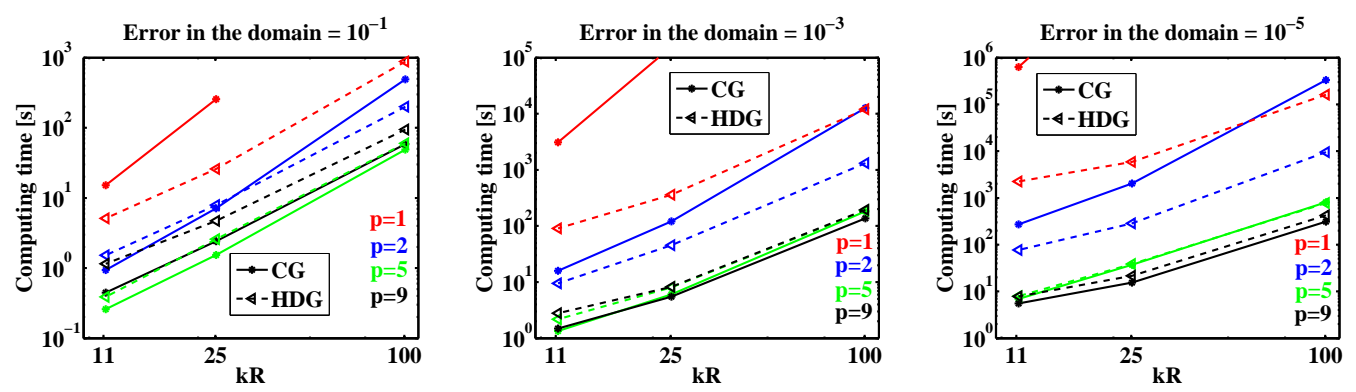

Figure 9. Circular scattering of a plane wave: runtime versus wavenumber at different precisions 1e-1 (left), 1e-3 (center), and 1e-5 (right).

In Figure 8 the relative $\mathcal{L}^{2}$ error in the domain is depicted as a function of the total computing time for $\mathrm{CG}$ and HDG, for $k R=11$ and $k R=100$. These results suggest that for a given accuracy CG is faster than HDG, at least for high-order approximations. To further analyze this issue and to confirm the better performance of high-order approximations, these curves, and also additional results with wave number $k=25$, are interpolated to obtain values of the computational time for fixed values of the error, as shown in Figure 9.

It is important to note that for a given accuracy, high-order elements require less computing time than low-order ones, also at low accuracy. Note also that high-order computations $p=5$ and $p=9$ induce, in general, a similar performance. For low precision $p=5$ outperforms $p=9$ but as the accuracy is tightened $p=9$ is faster than $p=5$. As expected, the best order $p$ depends on the actual problem to be solved. But, as a general rule, high-order elements $(p>2)$ are more efficient than low order ones, clearly outperforming the usual practice of linear approximations.

With respect to CG and HDG several observation can be extracted. Obviously, for $p=1$, HDG outperforms CG because no stabilization is used in CG whereas HDG incorporates it intrinsically. For $p=2$, except for low accuracies and large wavelengths where both methods are almost equivalent, $\mathrm{HDG}$ is faster than CG for given precision and this improvement of runtime in HDG is more important as $k$ increases. This is not the case for high-order approximations. For $p=5$ and 9 at a given precision, CG is faster than HDG. This is more obvious for low accuracies. As the precision increases both performances are similar.

In fact, it is worth noting that the overhead of HDG for a given discretization (given $p$ and $h$ ) has a simple behavior. Figure 10 clearly shows that the runtime overhead for a fixed mesh is almost independent of the characteristic element size and that it decreases as the degree of the approximation increases. For high degrees this overhead is almost constant for $p$. Consequently, for a given high-order CG discretization with a uniform characteristic mesh size, from Figure 10 and the Remark 5, one can compute the characteristic size of HDG inducing a similar computational cost.

Note however, as observed earlier, that the HDG overhead can be compensated at mid to high accuracies by its increased precision. In fact, recall that as the precision increases CG and HDG show similar performances.

Remark 5 (Runtime). Numerical evidence, as shown in this example, indicates that for CG and HDG with meshes having a number of boundary faces negligible compared to the number of interior ones, i.e. boundary influence is negligible, the runtime is approximately $\mathcal{O}\left(\mathrm{n}_{\mathrm{el}}\right) \approx \mathcal{O}\left(h^{-2}\right)$.

To further analyze the overhead of HDG respect to CG for a fixed mesh, Figure 11 shows the runtime ratio between HDG and CG. The left figure shows the ratio only for the linear system solve and the right one the total runtime ratio, i.e. linear solve plus all element computations: creation and assembly of matrices, element-by-element solution - Schur complement for CG and local solve for HDG, i.e. Eqs. (7a) - and post-process for HDG. Given the relative independence of the runtime overheads on the mesh size shown in Figure 10, Figure 11 shows averages at each degree $p$ for every computed mesh size $h$. 


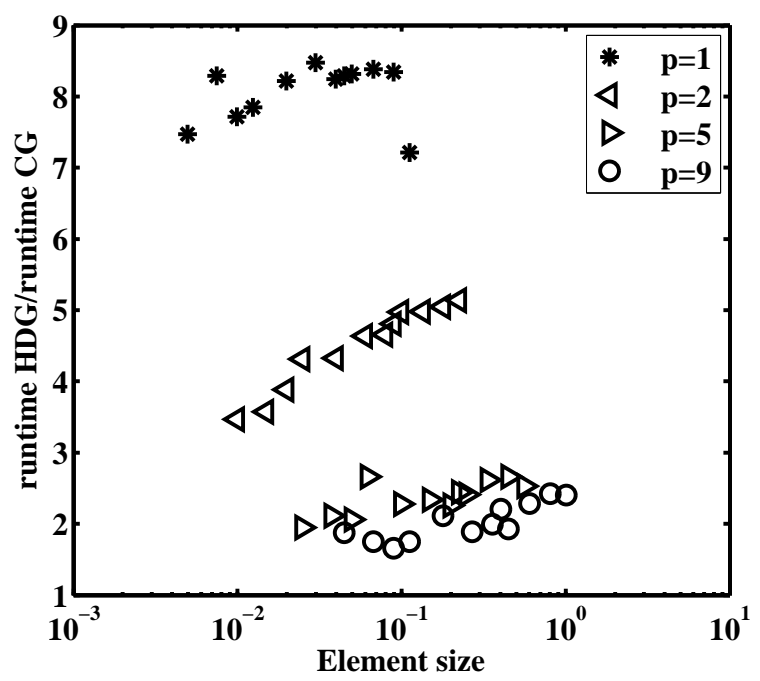

Figure 10. Circular scattering of a plane wave: runtime ratio between HDG and CG for fixed meshes.
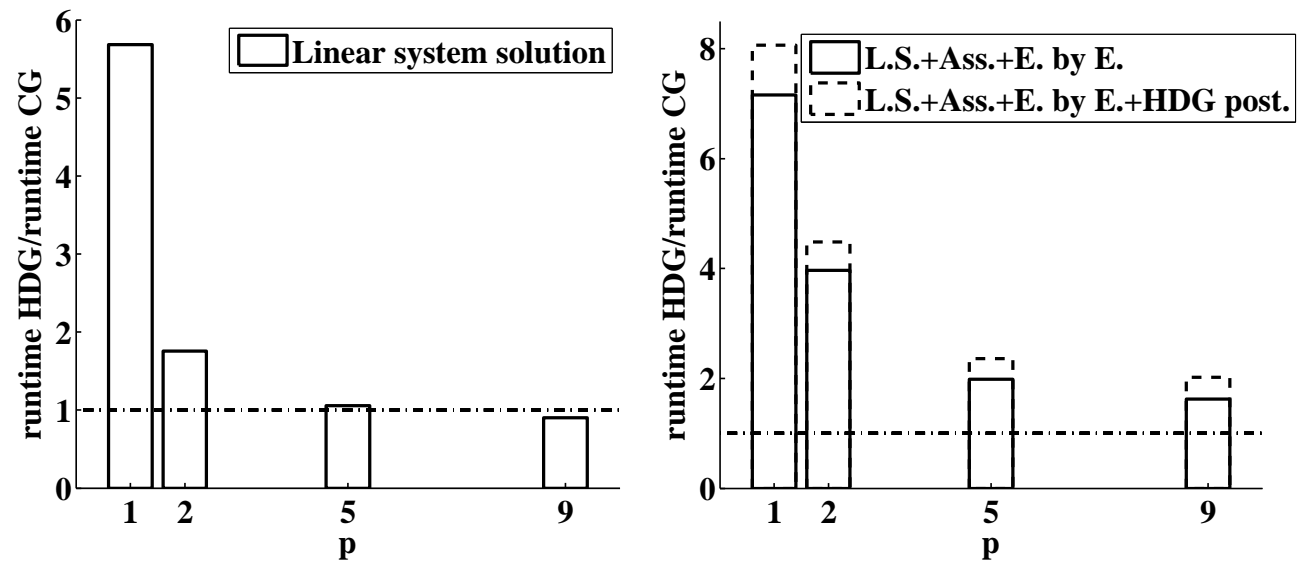

Figure 11. Circular scattering of a plane wave: runtime ratio between HDG and CG for fixed meshes only for the linear solve (left) and accounting for all element computations (right).

The remarkable overhead of HDG for linear elements compared to CG is drastically reduced as $p$ increases. In fact, in agreement with [41], HDG requires less runtime to solve the global linear system for high-orders. This is due to the uniform block-structure of HDG as discussed in Remark 2.

The local (elemental) computations, on the other hand, are always more costly for HDG, due its increased complexity in the local problems. This is shown in Figure 11 (right). In spite of its faster linear solve, HDG is still more expensive than $\mathrm{CG}$ even for $p=9$ when the total cost is considered. That is, adding to the global linear solve the time required for the assembly and the local element-byelement operations. Note however, all elemental computations are likely to be parallelized, reducing the weight of the elemental computations on the global solving time.

Finally, Figure 11 (right) also depicts the overhead associated to the post-process of the HDG solution which allows to compute a super-convergent approximation. This is to show that the extra cost is minor compared to the advantage of obtaining a solution with a higher precision or, simply, an estimation of the error. 

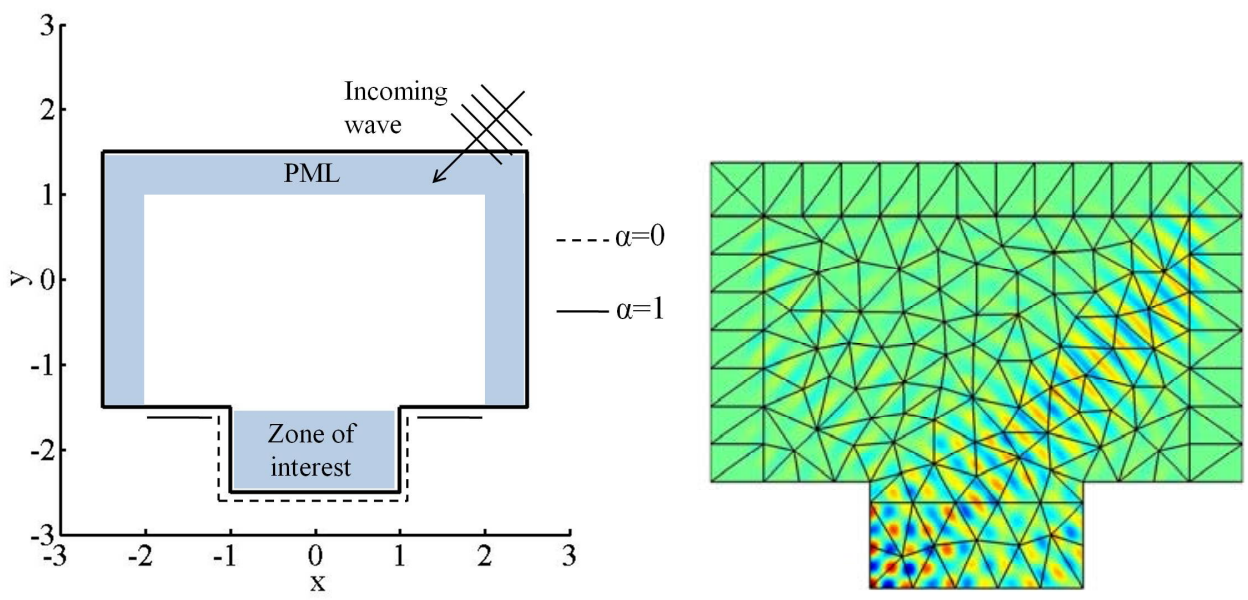

Figure 12. Rectangular cavity: problem statement (left) and scattered wave (right) on a mesh with $h=0.4$, $p=8$, and $\xi=1.26$.

\subsection{Rectangular cavity problem}

In this dimensionless example an incoming plane wave at angle $225^{\circ}$ with respect to the $x$-axis and a wavelength of 0.25 is propagated in a semi-plane with a squared notch. As in the previous example, the scattered wave is the solution of equation (4) with constant coefficient $\beta$ and $f=0$. The value of the absorption parameter used to model the boundary is $\alpha=0$ inside the notch (i.e. totally reflecting) and $\alpha=1$ (absorbing) on rest of the physical boundary. Figure 12 shows the problem statement and the scattered wave. The geometry is similar to the one used in [64] and ideally models the resonance of sea waves in a rectangular harbor with constant bathymetry. The rectangular closed region is considered as the zone of interest of the domain, where wall reflections generate a complex wave interaction pattern that makes the test particularly challenging from a computational point of view. In addition, as usually in the presence of non-smooth boundaries such as corners, the solution of the wave equation is singular leading to non-optimal convergence both for $h$ and $p$-refinement, see for instance $[44,45]$. This test aims to show that, in the absence of adaptive mesh refinement, high-order elements are more efficient than low-order ones, even in the presence of singularities.

Four different polynomial degree are tested, $p=1,2,4$, and 8 . For each degree four nested meshes are used, they correspond to $\xi=1.257,0.628,0.314$, and 0.157 (i.e. $5,10,20$, and 40 nodes per wavelength). Given that no analytical solution is available for this test, the relative $\mathcal{L}^{2}$ error in the zone of interest is evaluated comparing the solution with a reference solution computed with CG with an $h$-refined high-order mesh, with 160 nodes per wavelength.

Figure 13 depicts for CG and HDG the relative $\mathcal{L}^{2}$ error as a function of the number of nodes per wavelength, i.e. the dimensionless wave number $\xi$ (left), and as a function of the computing time (right). In the presence of singularities, as expected, all curves asymptotically reach the same slope [45] as the dimensionless wave number decreases (as the number of nodes per wavelength increases). In any case, high-order elements require less nodes per wavelength than low-order elements for a given accuracy. Thus, high-order approximations require less DOF for a given accuracy. This has a direct impact in the computing time as shown also in Figure 13 and, for a given accuracy, high-order elements are faster than low-order ones. Thus, it is worth noting that, in spite of the singularities, high-order approximations outperform low-order ones. Obviously, an $h p$ strategy would improve the performances, but this is out of the scope of the present comparison.

The performance of CG for $p=1$ is clearly hampered by the lack of stabilization. The comparison between CG and HDG should be focused on the other orders. For $p=2$ except for very low accuracies (below 0.1) HDG outperforms CG in runtime. Nonetheless, CG proves to be faster than HDG for a given accuracy for high-order elements. Obviously, the overhead of HDG decreases as the accuracy increases. 

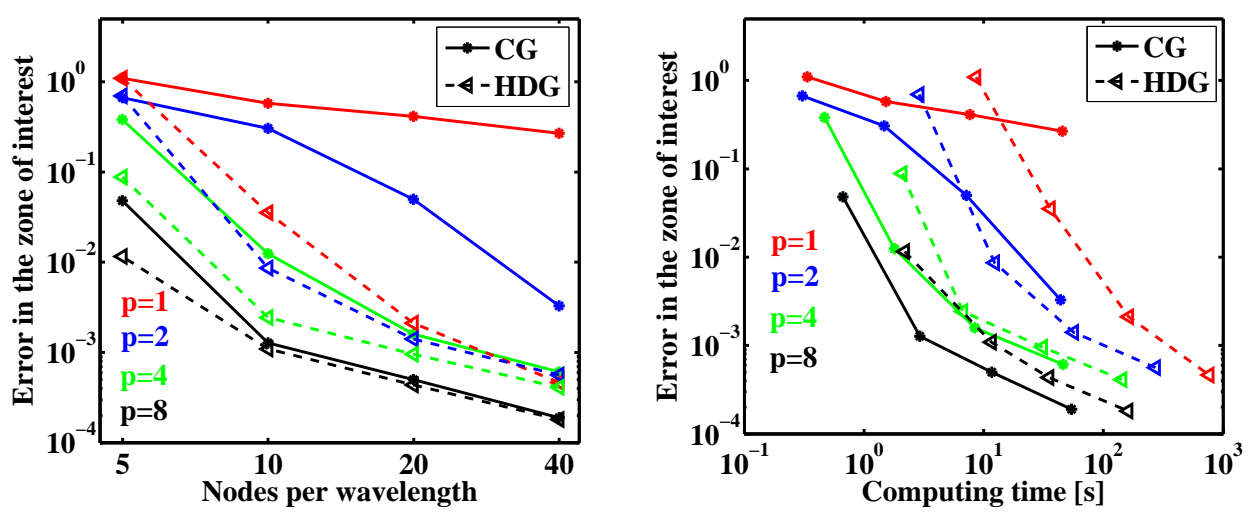

Figure 13. Rectangular cavity: error versus nodes per wavelength (left) and runtime (rigth).

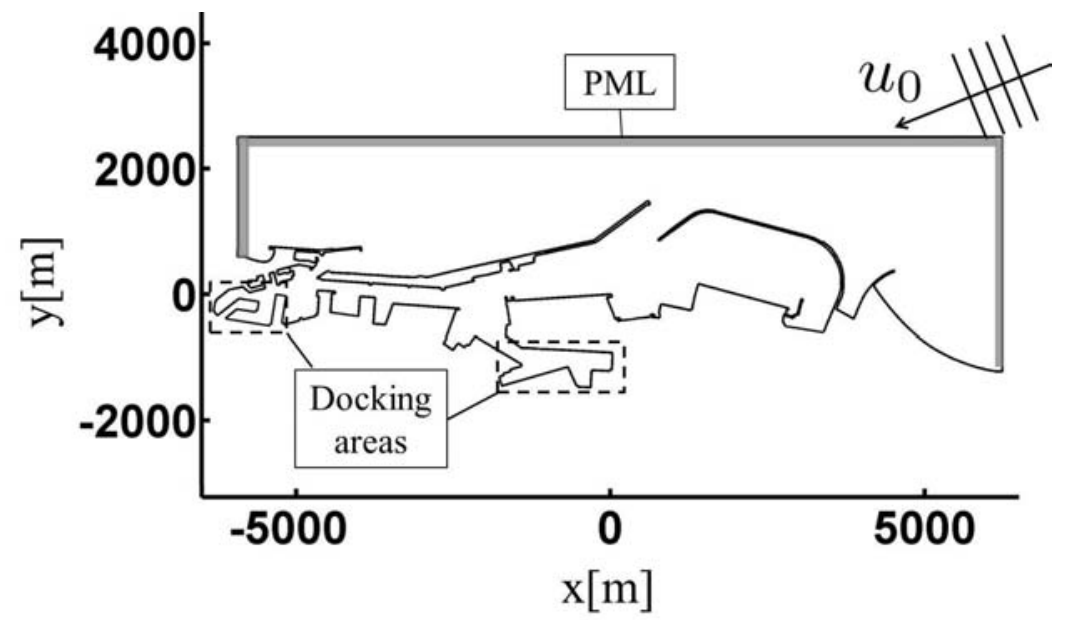

Figure 14. Barcelona harbor: statement of the problem.

\subsection{Barcelona harbor}

In this section, the Mild Slope Equation (MSE) $[65,66]$ is used to study the wave propagation in Barcelona's harbor. Thus, in this example, the coefficient $\beta$ in problem (4) is non-constant and depends on the bathymetry, see [65] for details. Moreover, the incident potential,

$$
u_{0}=\exp \left(i k_{0}\left(x \cos \theta_{0}+y \sin \theta_{0}\right)\right),
$$

with $k_{0}=0.1121$ (corresponding to a wavelength of $56 \mathrm{~m}$ ) and angle of incidence $\theta_{0}=202.4^{\circ}$ from the $x$-axis, induces a non-homogeneous equation, namely

$$
f=-\nabla \cdot\left(\beta \nabla u_{0}\right)-k^{2} \beta u_{0} .
$$

Figure 14 shows the computational domain, it ranges in latitude between $41.28^{\circ} \mathrm{N}$ and $41.38^{\circ} \mathrm{N}$ and covers an area of about $34.4 \mathrm{~km}^{2}$. The PML area is also depicted as well as the areas of interest. The bathymetry data comes from a real measure campaign ${ }^{\dagger}$, and it has been modified to comply the condition of constant bottom depth in the PML area. This condition is sufficient to ensure absorption of the PML media, see for example [67]. The absorption coefficient $\alpha$ on physical boundaries has been set to 0.05 for dikes, 0.43 for breakwaters and 0.74 for beaches. 


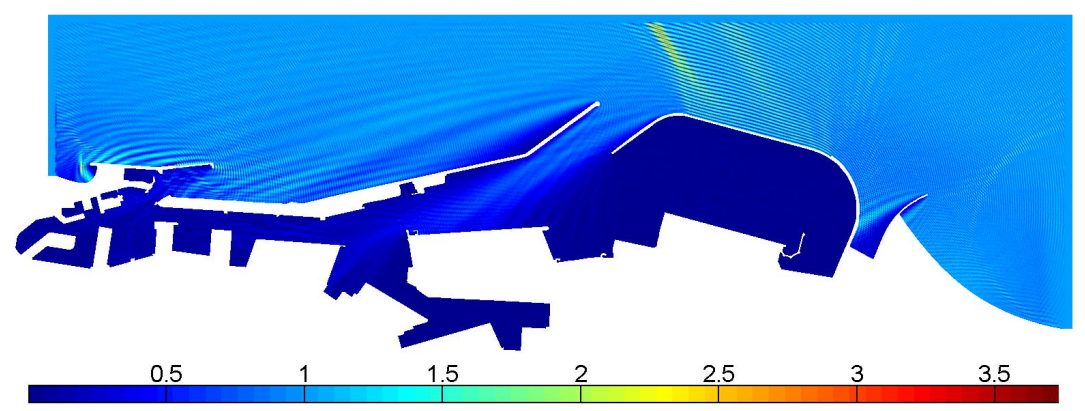

Figure 15. Barcelona harbor: wave amplification factor.
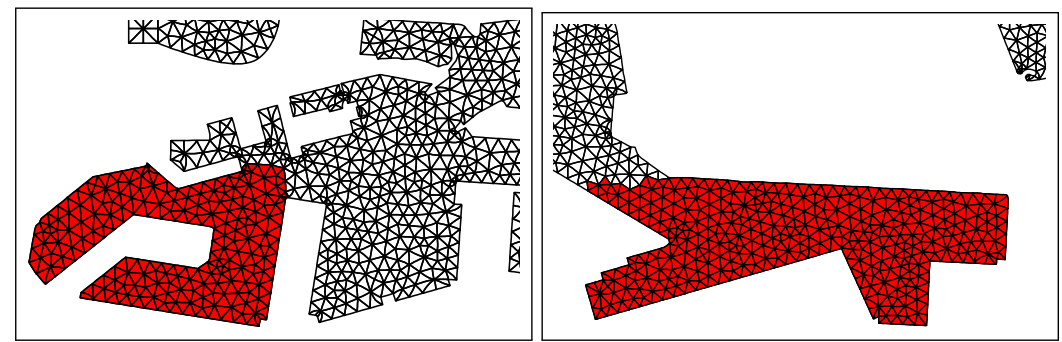

Figure 16. Barcelona harbor: zoom in the docking areas.

Table II. Barcelona harbor: error, DOF and runtime for CG.

\begin{tabular}{rrrrrrr}
\hline$h[\mathrm{~m}]$ & 4 & 10 & 10 & 50 & 50 & 50 \\
\hline$p$ & 2 & 2 & 4 & 8 & 10 & 12 \\
\hline error & $0.28 \mathrm{e}-2$ & $0.26 \mathrm{e}-1$ & $0.28 \mathrm{e}-3$ & $0.14 \mathrm{e}-1$ & $0.21 \mathrm{e}-2$ & $0.50 \mathrm{e}-3$ \\
max el.error & $0.16 \mathrm{e}-1$ & $0.14 \mathrm{e} 0$ & $0.15 \mathrm{e}-2$ & $0.38 \mathrm{e}-1$ & $0.87 \mathrm{e}-2$ & $0.22 \mathrm{e}-2$ \\
DOF & 4908818 & 1647698 & 4115482 & 367198 & 467198 & 567198 \\
runtime [s] & 318 & 80 & 515 & 79 & 168 & 485 \\
\hline
\end{tabular}

The usual output of interest for this problem is the so-called wave amplification factor, that is, the ratio between the total and incident wave-height, namely

$$
H=\frac{\left|u+u_{0}\right|}{\left|u_{0}\right|} \text {. }
$$

It is plotted for this case in Figure 15. The areas of interest, denoted as $\Omega_{\text {int }}$, already shown in Figure 14 are zoomed in Figure 16. The error in $\Omega_{\text {int }}$ is computed using a reference solution. Two measures of the error are considered, that is, the mean error

$$
E^{2}=\frac{1}{\operatorname{meas}\left(\Omega_{\text {int }}\right)} \int_{\Omega_{\text {int }}}\left(H^{*}-H\right)^{2} d \Omega
$$

and the maximum elemental error, that is

$$
E^{2}=\max _{\forall \Omega_{i} \in \Omega_{\text {int }}} \frac{1}{\operatorname{meas}\left(\Omega_{i}\right)} \int_{\Omega_{i}}\left(H^{*}-H\right)^{2} d \Omega
$$

where $H^{*}$ is the wave amplification factor computed with the reference solution.

Tables II and III summarize different indicators (mean error in the area of interest, maximum elemental error in the area of interest, dimension of the global system, and runtime) for CG and HDG and different discretizations. Three computational meshes with characteristic element size $h=4 \mathrm{~m}$, 
Table III. Barcelona harbor: error, DOF and runtime for HDG.

\begin{tabular}{rrrrrrrr}
\hline$h[\mathrm{~m}]$ & 4 & 10 & 10 & 10 & 50 & 50 & 50 \\
\hline$p$ & 2 & 1 & 2 & 4 & 8 & 10 & 12 \\
\hline error & $0.78 \mathrm{e}-4$ & $0.33 \mathrm{e}-1$ & $0.79 \mathrm{e}-3$ & $0.23 \mathrm{e}-3$ & $0.30 \mathrm{e}-2$ & $0.57 \mathrm{e}-3$ & $0.29 \mathrm{e}-3$ \\
max el.error & $0.98 \mathrm{e}-3$ & $0.13 \mathrm{e} 0$ & $0.35 \mathrm{e}-2$ & $0.95 \mathrm{e}-3$ & $0.95 \mathrm{e}-2$ & $0.30 \mathrm{e}-2$ & $0.16 \mathrm{e}-2$ \\
DOF & 11033058 & 2467784 & 3701676 & 6169460 & 450000 & 550000 & 650000 \\
runtime [s] & 2426 & 435 & 671 & 1722 & 224 & 486 & 1125 \\
\hline
\end{tabular}

10m, and 50m have been considered, with 2446554 , 820086 , and 32802 elements, respectively. Obviously, smaller elements are present in the interior of the harbor to properly capture geometric details. As an example, Figure 16 also shows in the area of interest the mesh of characteristic size $h=50 \mathrm{~m}$. For this mesh, the smoothing technique proposed in [68] has been used for untangle elements and improve the quality of the mesh.

It is worth noting that the wave number depends on the bottom depth through the so-called dispersion relation, leading to a non-uniform dimensionless wave number $\xi$, see Remark 4 , for a fixed element size $h$. As an example, the dimensionless wave number for the discretization with characteristic element size $h=10 \mathrm{~m}$ could vary for a degree $p=10$ from $\xi=0.2$ (30 nodes per wavelength) in the docking areas with bottom depth of about $3 \mathrm{~m}$, to $\xi=0.11$ (57 nodes per wavelength) in open sea with bottom depth of $30 \mathrm{~m}$.

As noted earlier, if runtime must be estimated the quadrature rule must determined. In this example, the coefficients are non-constant. Using quadratures (with the minimum number of integration points) from Table I able to integrate exactly polynomials up to degree $3 p$, gives sufficient resolution and allows a fair comparison of runtime between CG and HDG.

Results in Tables II and III, corroborate that both CG and HDG perform better with large highorder elements than smaller low-order ones. As in the previous examples, the use of large high-order elements reduces the computational cost for a given level of accuracy.

For instance, comparing CG with $\{h=4, p=2\}$ and $\{h=50, p=10\}$ it can be noticed that both have a similar mean error in the area of interest but $\{h=4, p=2\}$ has a larger maximum error, ten times more DOF and twice runtime. High-order methods also perform better for lower accuracies, one order lower when $\{h=10, p=2\}$ is compared with $\{h=50, p=8\}$ in the same table. In this case the runtime is similar but the high-order approximation induces smaller errors, in particular, the maximum one.

Table III corroborates the same conclusions for HDG. For instance, HDG solutions with $\{h=$ $10, p=2\}$ and $\{h=50, p=10\}$ have similar accuracies (in terms of mean or maximum error) but the high-order approach is 1.4 times faster. When comparing $\{h=10, p=4\}$ and $\{h=50, p=12\}$ the ratio is also similar, 1.5 time faster the high-order approximation.

Note also that, as expected, the comparison of Tables II and III shows once again how HDG provides better accuracy than CG for the same computational mesh, but with an overhead in runtime, which decreases as the degree of approximation increases (recall Figure 11). This motivates to compute the characteristic size of HDG inducing a similar computational cost for a given highorder CG discretization, as stated in Section 4.1. Table IV shows precisely the results of CG for $h=50$ and those of HDG on a mesh with a characteristic size $h=84$, which is the value obtained from Figure 10 and Remark 5. Consequently, the computational costs displayed in Table IV are similar for CG and HDG. This table confirms that, for a similar computational cost, CG and HDG have similar accuracy. Nevertheless, $p$-adaptive strategies are more easily implemented in an HDG framework [69] than in a CG code. 
Table IV. Barcelona harbor: analogous CG and HDG results.

\begin{tabular}{rrrrr}
\hline & $p$ & 8 & 10 & 12 \\
\hline & error & $0.14 \mathrm{e}-1$ & $0.21 \mathrm{e}-2$ & $0.50 \mathrm{e}-3$ \\
CG with $h=50 \mathrm{~m}$ & max el. error & $0.38 \mathrm{e}-1$ & $0.87 \mathrm{e}-2$ & $0.22 \mathrm{e}-2$ \\
& DOF & 367198 & 467198 & 567198 \\
& runtime [s] & 79 & 168 & 485 \\
\hline \multirow{3}{*}{ HDG with $h=84 \mathrm{~m}$} & error & $0.13 \mathrm{e}-1$ & $0.11 \mathrm{e}-2$ & $0.70 \mathrm{e}-3$ \\
& max el. error & $0.60 \mathrm{e}-1$ & $0.44 \mathrm{e}-2$ & $0.25 \mathrm{e}-2$ \\
& DOF & 164259 & 200761 & 237263 \\
& runtime [s] & 82 & 175 & 408 \\
\hline
\end{tabular}

\section{CONCLUDING REMARKS}

High and low-order, continuous and discontinuous, finite elements are compared for 2D scattering problems in unbounded domains. Moreover, non-constant coefficients are also considered. This is typical of the Mild Slope Equation for modeling the propagation of sea waves in harbors. In fact, this is one of the three numerical examples used to evaluate the performance of the different approaches in terms of accuracy, dimension of the global linear system (number of DOF) and runtime.

For this wave propagation problem, high-order approximations based on polynomial approximations outperform low-order approaches for a wide range of wavelengths, in particular, from low to mid-high frequencies. They require less degrees of freedom for a given accuracy and, consequently, less computational time. This is also the case in examples with singularities and holds both for continuous and discontinuous methods.

Two DG methods are compared with the standard continuous Galerkin (CG) approach: the Compact Discontinuous Galerkin (CDG) method, as a representative DG method with compact stencil, and the Hybridizable Discontinuous Galerkin (HDG) method. HDG induces an important reduction in number of DOF for high-order elements. CG and HDG exhibit comparable efficiency, providing similar levels of accuracy for the same computational cost, and clearly outperforming CDG. Note however that HDG has more degrees of freedom (this overhead decreases as the order of approximation is increased) and is more accurate than CG on the same discretization. However, it is also show that the uniform block structure of HDG allows better computational efficiently compared to CG.

\section{ACKNOWLEDGEMENT}

Bathymetry data for the Barcelona harbor comes from a real campaign of measure courtesy of the Autoritat Portuaria de Barcelona.

\section{REFERENCES}

1. Thompson LL, Pinsky PM. Complex wavenumber Fourier analysis of the $p$-version finite element method. Comput. Mech. 1994; 13(4):255-275, doi:10.1007/BF00350228.

2. Ihlenburg F, Babuška I. Finite element solution of the Helmholtz equation with high wave number Part I: The h-version of the FEM. Comput. Math. Appl. 1995; 30(9):9-37.

3. Ihlenburg F, Babuška I. Finite element solution of the Helmholtz equation with high wave number Part II: The h-p version of the FEM. SIAM J. Numer. Anal. 1997; 34(1):315-358.

4. Steffens LM, Díez P. A simple strategy to assess the error in the numerical wave number of the finite element solution of the Helmholtz equation. Comput. Methods Appl. Mech. Eng. 2009; 198(15-16):1389-1400.

5. Steffens LM, Parés N, Díez P. Estimation of the dispersion error in the numerical wave number of standard and stabilized finite element approximations of the Helmholtz equation. Int. J. Numer. Methods Eng. 2011; 86(10):11971224.

6. Chang C. A least-squares finite element method for the Helmholtz equation. Comput. Methods Appl. Mech. Eng. 1990; 83(1):1-7. 
7. Harari I, Hughes TJR. Galerkin/least-squares finite element methods for the reduced wave equation with nonreflecting boundary conditions in unbounded domains. Comput. Methods Appl. Mech. Eng. 1992; 98(3):411454.

8. Babuška I, Ihlenburg F, Paik ET, Sauter SA. A generalized finite element method for solving the Helmholtz equation in two dimensions with minimal pollution. Comput. Meth. Appl. Mech. Eng. 1995; 128(3-4):325-359.

9. Harari I, Avraham D. High-order finite element methods for acoustic problems. J. Comput. Acoust. 1997; $\mathbf{0 5}(01): 33-51$.

10. Franca LP, Farhat C, Macedo AP, Lesoinne M. Residual-free bubbles for the Helmholtz equation. Int. J. Numer. Methods Eng. 1997; 40(21):4003-4009.

11. Babuška I, Sauter SA. Is the pollution effect of the FEM avoidable for the Helmholtz equation considering high wave numbers? SIAM Rev. 2000; 42(3):451-484.

12. Harari I, Magoulès F. Numerical investigations of stabilized finite element computations for acoustics. Wave Motion 2004; 39(4):339-349.

13. Harari I, Tezaur R, Farhat C. A study of higher-order discontinuous Galerkin and quadratic least-squares stabilized finite element computations for acoustics. J. Comput. Acoust. 2006; 14(1):1-19.

14. Harari I, Gosteev K. Bubble-based stabilization for the Helmholtz equation. Int. J. Numer. Methods Eng. 2007; 70(10): 1241-1260.

15. Loula AFD, Fernandes DT. A quasi optimal Petrov-Galerkin method for Helmholtz problem. Int. J. Numer. Methods Eng. 2009; 80(12):1595-1622.

16. Melenk J, Babuška I. The partition of unity finite element method: Basic theory and applications. Comput. Meth. Appl. Mech. Eng. 1996; 139(1-4):289-314.

17. Cessenat O, Despres B. Application of an ultra weak variational formulation of elliptic PDEs to the two-dimensional Helmholtz problem. SIAM J. Numer. Anal. 1998; 35(1):255-299.

18. Lacroix V, Bouillard P, Villon P. An iterative defect-correction type meshless method for acoustics. Int. J. Numer. Methods Eng. 2003; 57(15):2131-2146.

19. Farhat C, Harari I, Hetmaniuk U. A discontinuous Galerkin method with Lagrange multipliers for the solution of Helmholtz problems in the mid-frequency regime. Comput. Methods Appl. Mech. Eng. 2003; 192(11-12):13891419.

20. Strouboulis T, Hidajat R. Partition of unity method for Helmholtz equation: q-convergence for plane-wave and wave-band local bases. Appl. Math. 2006; 51(2):181-204.

21. Gabard G. Discontinuous Galerkin methods with plane waves for time-harmonic problems. J. Comput. Phys. 2007; 225(2): 1961-1984.

22. Gabard G, Gamallo P, Huttunen T. A comparison of wave-based discontinuous Galerkin, ultra-weak and leastsquare methods for wave problems. Int. J. Numer. Methods Eng. 2011; 85(3):380-402.

23. Wang D, Tezaur R, Toivanen J, Farhat C. Overview of the discontinuous enrichment method, the ultra-weak variational formulation, and the partition of unity method for acoustic scattering in the medium frequency regime and performance comparisons. Int. J. Numer. Methods Eng. 2011; 89(4):403-417.

24. Ihlenburg F, Babuška I. Dispersion analysis and error estimation of Galerkin finite element methods for the Helmholtz equation. Int. J. Numer. Methods Eng. 1995; 38(22):3745-3774.

25. Vos PEJ, Sherwin SJ, Kirby RM. From $h$ to $p$ efficiently: implementing finite and spectral $/ h p$ element methods to achieve optimal performance for low- and high-order discretisations. J. Comput. Phys. 2010; 229(13):5161-5181.

26. Cantwell CD, Sherwin SJ, Kirby RM, Kelly PHJ. From $h$ to $p$ efficiently: selecting the optimal spectral/hp discretisation in three dimensions. Math. Model. Nat. Phenom. 2011; 6(3):84-96.

27. Huerta A, Roca X, Angeloski A, Peraire J. Are High-order and Hybridizable Discontinuous Galerkin methods competitive? Oberwolfach Rep. 2012; 9(1):485-487, doi:10.4171/OWR/2012/09. Abstracts from the workshop held February 12-18, 2012, Organized by Olivier Allix, Carsten Carstensen, Jörg Schröder and Peter Wriggers, Oberwolfach Reports. Vol. 9, no. 1.

28. Modesto D, Giorgiani G, Zlotnik S, Huerta A. Efficiency and accuracy of high-order computations and reduced order modelling in coastal engineering wave propagation problems. Proceedings of the ECCOMAS Young Investigators Conferences 2012, Aveiro, Portugal, 2012.

29. Sevilla R, Hassan O, Morgan K. An analysis of the performance of a high-order stabilised finite element method for simulating compressible flows. Comput. Methods Appl. Mech. Eng. 2013; 253(0):15-27, doi:10.1016/j.cma.2012. 09.001.

30. Giorgiani G, Fernández-Méndez S, Huerta A. Hybridizable Discontinuous Galerkin p-adaptivity for wave propagation problems. Int. J. Numer. Methods Fluids 2013; doi:10.1002/fld.3784. To appear.

31. Huerta A, Angeloski A, Roca X, Peraire J. Efficiency of high-order elements for continuous and discontinuous galerkin methods. Int. J. Numer. Methods Eng. 2013; To appear.

32. Arnold DN. An interior penalty finite element method with discontinuous elements. SIAM J. Numer. Anal. 1982; 19(4):742-760.

33. Arnold DN, Brezzi F, Cockburn B, Marini LD. Unified analysis of discontinuous Galerkin methods for elliptic problems. SIAM J. Numer. Anal. 2001; 39(5):1749-1779.

34. Peraire J, Persson PO. The compact discontinuous Galerkin (CDG) method for elliptic problems. SIAM J. Sci. Comput. 2008; 30(4):1806-1824.

35. Cockburn B, Dong B, Guzmán J. A superconvergent LDG-hybridizable Galerkin method for second-order elliptic problems. Math. Comp. 2008; 77(264):1887-1916.

36. Nguyen NC, Peraire J, Cockburn B. An implicit high-order hybridizable discontinuous Galerkin method for linear convection-diffusion equations. J. Comput. Phys. 2009; 228(9):3232-3254.

37. Nguyen N, Peraire J, Cockburn B. A hybridizable discontinuous Galerkin method for Stokes flow. Comput. Methods Appl. Mech. Eng. 2010; 199(9-12):582-597.

38. Feng X, Wu H. Discontinuous Galerkin methods for the Helmholtz equation with large wave number. SIAM J. Sci. Comput. 2009; 47(4):2872-2896. 
39. Alvarez GB, Loula AFD, do Carmo EGD, Rochinha FA. A discontinuous finite element formulation for Helmholtz equation. Comput. Methods Appl. Mech. Eng. 2006; 195(33-36):4018-4035.

40. Cockburn B, Gopalakrishnan J, Lazarov R. Unified hybridization of discontinuous Galerkin, mixed, and continuous Galerkin methods for second order elliptic problems. SIAM J. Numer. Anal. 2009; 47(2):1319-1365.

41. Kirby R, Sherwin S, Cockburn B. To CG or to HDG: A comparative study. J. Sci. Comput. 2011; 51(1):183-212.

42. Roca X, Nguyen NC, Peraire J, Huerta A. A GPU-accelerated iterative solver for a hybridized discontinuous Galerkin method. Proceedings of the 23rd International Conference on Parallel Computational Fluid Dynamics, Barcelona, Spain, 2011.

43. Roca X, Nguyen NC, Peraire J. Implementing a hybridized discontinuous Galerkin solver on the GPU using orderoblivious indexing. Proceedings of the 16th International Conference on Finite Elements in Flow Problems (FEF 2011), International Association for Computational Mechanics (IACM): Munich, Germany, 2011.

44. Costabel M, Dauge M. Singularities of electromagnetic fields in polyhedral domains. Arch. Ration. Mech. Anal. 2000; 151:221-276.

45. Babuška I, Szabo B. Finite element analysis. John Wiley \& Sons: New York, 1991.

46. Berkhoff J. Mathematical models for simple harmonic linear water waves: wave diffraction and refraction. PhD Thesis, Delft Hydraulics Laboratory, Delft University of Technology, Delft, Netherlands 1976.

47. Givoli D. Numerical methods for problems in infinite domains, Studies in Applied Mechanics, vol. 33. Elsevier Scientific Publishing Co.: Amsterdam, 1992.

48. Givoli D, Neta B. High-order non-reflecting boundary scheme for time-dependent waves. J. Comput. Phys. 2003; 186(1):24-46.

49. Abarbanel S, Gottlieb D. On the construction of absorbing layers in CEM. Appl. Numer. Math. 1998; 27(4):331340 .

50. Berenger JP. A perfectly matched layer for the absorption of electromagnetic waves. J. Comput. Phys. 1994; 114(2):185-200.

51. Turkel E, Yefet A. Absorbing PML boundary layers for wave-like equations. Appl. Numer. Math. 1998; 27(4):533557.

52. Bermúdez A, Hervella-Nieto L, Prieto A, Rodríguez R. An exact bounded PML for the Helmholtz equation. $C$. $R$. Math. 2004; 339(11):803-808.

53. Taylor MA, Wingate BA, Vincent RE. An algorithm for computing Fekete points in the triangle. SIAM J. Numer. Anal 2000; 38:1707-1720.

54. Sevilla R, Fernandez-Mendez S, Huerta A. NURBS-Enhanced Finite Element Method (NEFEM) a seamless bridge between CAD and FEM. Arch. Comput. Method Eng. 2011; 18(4):441-484.

55. Montlaur A, Fernández-Méndez S, Huerta A. Discontinuous Galerkin methods for the Stokes equations using divergence-free approximations. Int. J. Numer. Methods Fluids 2008; 57(9):1071-1092.

56. Cockburn B, Kanschat G, Perugia I, Schotzau D. Superconvergence of the local discontinuous Galerkin method for elliptic problems on cartesian grids. SIAM J. Numer. Anal. 2002; 39(1):264-285.

57. Montlaur A, Fernández-Méndez S, Peraire J, Huerta A. Discontinuous Galerkin methods for the Navier-Stokes equations using solenoidal approximations. Int. J. Numer. Methods Fluidss 2010; 64(5):549-564.

58. Cockburn B, Guzmán J, Wang H. Superconvergent discontinuous Galerkin methods for second-order elliptic problems. Math. Comput. 2009; 78:1-24.

59. Wandzurat S, Xiao H. Symmetric quadrature rules on a triangle. Comput. Math. Appl. 2003; 45(12):1829-1840.

60. Dunavant DA. High degree efficient symmetrical Gaussian quadrature rules for the triangle. Int. J. Numer. Methods Eng. 1985; 21(6):1129-1148.

61. Donea J, Huerta A. Finite element methods for flow problems. John Wiley \& Sons: Chichester (UK), 2003.

62. Bowman JJ, Senior TB, Uslenghi PLE. Electromagnetic and Acoustic Scattering by Simple Shapes. Hemisphere: New York, 1987.

63. MacCamy RC, Fuchs RA. Wave forces on piles: a diffraction theorys. Technical Report, US Army Corps of Engineering, Beach Erosion Board, Washington, DC 1954.

64. Panchang VG, Pearce BR, Wei G, Cushman-Roisin B. Solution of the mild-slope wave problem by iteration. Appl. Ocean Res. 1991; 13(4):187-199.

65. Berkhoff JCW. Computation of combined refraction-diffraction. Proceedings of the 13th Coastal Engineering Conference, vol. 1, ASCE: Vancouver, Canada, 1972; 471-490.

66. Booij N. A note on the accuracy of the mild-slope equation. Coast. Eng. 1983; 7(3):191-203.

67. Kucukcoban S, Kallivokas L. Mixed perfectly-matched-layers for direct transient analysis in 2D elastic heterogeneous media. Comput. Meth. Appl. Mech. Eng. 2011; 200(1-4):57-76.

68. Roca X, Gargallo-Peiró A, Sarrate J. Defining quality measures for high-order planar triangles and curved mesh generation. Proceedings of the 20th International Meshing Roundtable, Paris, France, 2011.

69. Giorgiani G, Fernández-Méndez S, Huerta A. Hybridizable discontinuous Galerkin p-adaptivity for wave problems. Proceedings of the ECCOMAS Young Investigators Conferences 2012, Aveiro, Portugal, 2012. 